\title{
Bioinspired Unidirectional Liquid Transport Micro-nano Structures: A Review
}

\author{
Liwen Zhang ${ }^{1 \dagger}$, Guang Liu ${ }^{1 \dagger}$, Huawei Chen ${ }^{12^{*}}$, Xiaolin Liu ${ }^{1}$, Tong Ran ${ }^{1}$, Yi Zhang ${ }^{1}$, \\ Yang Gan ${ }^{1}$, Deyuan Zhang ${ }^{1,2}$ \\ 1. School of Mechanical Engineering and Automation, Beihang University, Beijing 100191, China \\ 2. Beijing Advanced Innovation Center for Biomedical Engineering, Beihang University, Beijing 100191, China
}

\begin{abstract}
Unidirectional liquid transport without any need of external energy has drawn worldwide attention for its potential applications in various fields such as microfluidics, biomedicine and mechanical engineering. In nature, numerous creatures have evolved such extraordinary unidirectional liquid transport ability, such as spider silk, Sarracenia's trichomes, and Nepenthes alata's peristome, etc. This review summarizes the current progresses of natural unidirectional liquid transport on 1-Dimensional (1D) linear structure and 2-Dimensional (2D) surface structure. The driving force of unidirectional liquid transport which is determined by unique structure exist distinct differences in physics. The fundamental understanding of 1D and 2D unidirectional liquid transport especially about hierarchical structural characteristics and their transport mechanism were concentrated, and various bioinspired fabrication methods are also introduced. The applications of bioinspired directional liquid transport are demonstrated especially in fields of microfluidics, biomedical devices and anti-icing surfaces. With newly developed smart materials, various liquid transport regulation strategies are also summarized for the control of transport speed, direction guiding, etc. Finally, we provide new insights and future perspectives of the directional transport materials.
\end{abstract}

Keywords: bioinspiration, unidirectional liquid transport, microfluidics, smart materials, Nepenthes alata, Sarracenia trichomes Copyright $($ C) The author(s) 2021.

\section{Introduction}

Unidirectional liquid transport has gradually attracted worldwide attention due to its diverse potential applications, such as water collection ${ }^{[1]}$, microflui$\operatorname{dics}^{[2,3]}$, lubrication ${ }^{[4-8]}$, anti-adhesion ${ }^{[9-12]}$ and liquid transportation $^{[13,14]}$. With millions of years of evolution, many nature plants have developed such functional structures or surfaces to directional transport water for survival without any need of external energy ${ }^{[15-25]}$. Generally, these natural surfaces or structures possess unique hierarchical micro-nano structures and various material wettability. Revealing the underlying mechanism of liquid directional transport could provide the fundamental understanding and creative inspiration to realize more complex liquid behavior regulations, such as ultra-fast transport, programable transport, and even micro/nanoscale liquid regulation.

Bioinspired hierarchical structure with unidirectional liquid transport can be classified into 1D-thread and 2D surface based on their structure types. 1D-thread structures that were found in spider silks ${ }^{[19]}$, cactus spines $^{[20]}$, and shorebird beaks ${ }^{[17]}$ make use of spindle knots wires, long sharp cones and wedged corner structures to directional harvest water from fog or suck water against gravity. Especially, the two-level hierarchical microgrooves on the conical trichomes of Sarracenia can directionally transport liquid from tip to root at the speed two orders higher than cactus spines ${ }^{[24]}$. The 2D surface is another type of unidirectional liquid transport that could perform continuous transport with a large volume of liquid. Desert beetle's back with patterned 2D surface wettability can directionally aggregate deposited water from the hydrophobic area to the hydrophilic area for feeding ${ }^{[15]}$. Nepenthes alata's peristome 2D surface with hierarchical microgrooves and duckbilled microcavities can continuously unidirectional transport liquid to form a slippery liquid film on peristome to capture insects ${ }^{[25]}$.

Over the past decades, the mechanisms of 1D and

\footnotetext{
*Corresponding author: Huawei Chen

E-mail: chenhw75@buaa.edu.cn

${ }^{\dagger}$ Liwen Zhang and Guang Liu contributed equally to this work.
} 
2D unidirectional liquid transport have been built from the coupling effect of feature microstructures and materials wettability. On 1D nature samples, the driving force of directional liquid transport abilities is mainly ascribed to an unbalanced capillary force formed by anisotropic linear structures, such as conical wire, conical tube, or wedged corner. On 2D unidirectional transport surface, periodic micro-nano structure array results in anisotropic liquid spreading and pinning effect to form a unidirectional transport with much higher speed and longer distance.

Significant progress of bioinspired 1D and 2D unidirectional liquid transport structures has been made, such as the bioinspired design of 1D directional transport with spindle knots wire, cone array surface, and hierarchical cones structure ${ }^{[26-28]}$, and 2D directional transport with inclined pillar arrays surface and $\mathrm{Ne}$ penthes peristome mimetic surface ${ }^{[29,30]}$. Various applications have also been realized in diverse fields, such as water collection, oil/water emulsion separation, microreactors, and microfluidics ${ }^{[31-33]}$. Moreover, with the new development of smart materials, the control of liquid transporting in 1D and 2D are presented by adjusting feature structures or surface wettability under the control of temperature, light, magnetism, etc ${ }^{[31,34,35]}$.

The purpose of this paper is to provide a summary of researches in bioinspired unidirectional liquid transport in 1D and 2D over the past decades. This review is composed of the illustration of two aspects based on 1D and 2D, where each aspect is organized with four parts, including a description of natural samples, underlying mechanisms, bioinspired design and fabrication, and potential applications. Finally, a perspective has been given for the future exploration of bioinspired unidirectional liquid transport.

\section{Different types of unidirectional liquid transport in nature}

\subsection{D unidirectional liquid transport in nature}

In nature, various samples with different surface structures and material properties exhibit 1D unidirectional liquid transporting ability, which can deposit water from fog or pump up water against gravity. When spider silks are placed in mist, the silk contracts under humidity and changes into a wire with periodically connected spindle knots and slender joints, where each slender joint and spindle knot creates a conical structure with gradually increased surface roughness and enlarged diameter from $41.66 \mu \mathrm{m} \pm 8.3 \mu \mathrm{m}$ to $130.86 \mu \mathrm{m} \pm$ $11.1 \mu \mathrm{m}^{[19]}$. With these conical structures, the deposited water droplets on slender joints could be intermittently and unidirectionally transported to spindle knots, then aggregates into a large water droplet (Fig. 1a). To effectively collect water in deserts, cactus evolves a hierarchical structure with long sharp conical spines and small conical barbs at the tip of these spines ${ }^{[20]}$. The fog firstly deposits on small conical barbs and directionally moves to barb root. With accumulated water from various barbs, a larger droplet appears at the tip of the conical spine and eventually directionally transported to the spine root for cactus's water harvesting. Apart from the directional flow of liquid on the outside surface of spider silk and cactus spine, shorebird beaks with a wedged shape corner can unidirectionally elevate water between its inner surfaces (Fig. 1b) ${ }^{[17]}$. For the directional liquid transport on spider silks, cactus spines, and shorebird beaks, their transporting speed normalized to the structure size is at tens to hundreds of unit length per second. To create a higher transporting speed, a novel surface liquid adjusting strategy is needed.

As a typical tropical plant, Sarracenia Sp.'s trichomes demonstrate a strong unidirectionally transporting ability with speed about two orders of magnitude faster than on the cactus spine (Fig. 1c) ${ }^{[24]}$. The trichome has a sharp conical shape with its surface covered by two-level high-low microgrooves. Its unidirectional liquid transport process can be divided into two modes, Mode I and Mode II. Mode I is similar to the water droplet transporting on spider silk and cactus spine, where deposited droplet directionally moves from trichome tip to root. Unlike the droplet moving entirely to root on spider silk and cactus spine, a thin water film is left on the trichome surface by the capillarity of microgrooves. With this thin water film, Mode II forms with directional liquid transport at an astonishingly ultrafast speed of $\sim 11738 \mu \mathrm{m} \cdot \mathrm{s}^{-1} \pm 715 \mu \mathrm{m} \cdot \mathrm{s}^{-1}$. Such unique conical structure with hierarchical microgrooves gives profound inspirations for bioinspired ultrafast 1D unidirectional liquid transport. The structural and material features of natural $1 \mathrm{D}$ unidirectional liquid transport are 

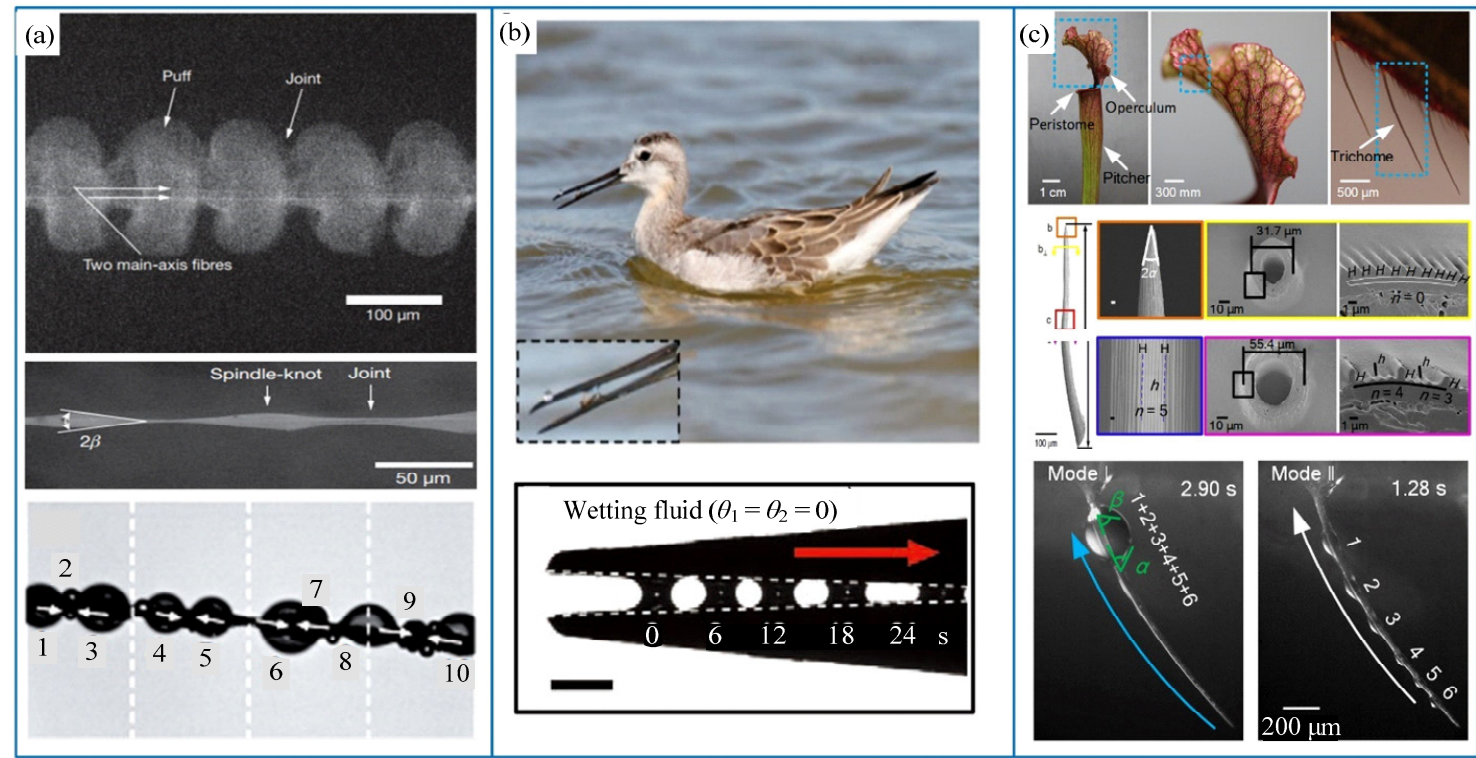

Fig. 1 (a) On spider silk, water droplets deposited from fog unidirectionally aggregate to spindle knots (reproduced from Ref. [19] with permission). (b) The wedged corner formed by shorebird beaks can directionally lift water against gravity for its water feeding (reproduced from Ref. [17] with permission). (c) With unique surface liquid adjusting effect, ultrahigh-speed liquid directional transport appears on Sarracenia trichomes (reproduced from Ref. [24] with permission).

listed in Table 1. Their anisotropic 1D structures like conical or wedged shape are critical for liquid directional transport, and the hierarchical micro-nano structures and materials characteristics covering the surface could also greatly affected its transporting performance.

\subsection{D unidirectional liquid transport in nature}

Liquid unidirectional transport on 2D surfaces is another interesting phenomenon in nature. Compared to 1D directional liquid transport in small and separated droplets, 2D directional transport on surfaces could have a continuous transport with a much higher volume transporting rate. Desert beetles, Stenocara $s p$., in Southern Africa can harvest fog with its back, which is covered by intermittent hydrophilic and hydrophobic areas. During the deposition, the fog droplets hit the hydrophobic area keep directionally flowing to the hydrophilic area (Fig. 2a), which finally aggregates into large droplets for its water feeding ${ }^{[15]}$. Compared to the gradient wettability on desert beetle's back, the butterfly wings with uniform material property has an asymmetric ratchet structure formed by micro-nano periodic stacked cuticle lamellae (Fig. 2b). The surface exhibits directional dependent water rolling-off angles, where coalesced droplets on wings are much easier to directionally roll to outward than to inward of wings ${ }^{[23]}$.

As a carnivorous plant, the peristome of Nepenthes is super slippery for insects capturing by covering a thin layer of water on the surface. To maintain such super slip, the peristome surface has evolved a strong unidirectional liquid transporting ability that can continuously transport liquid from the peristome inside to outside with speed up to $\sim 78 \mathrm{~mm} \cdot \mathrm{s}^{-1}$ (Fig. 2c) ${ }^{[25]}$. The surface is uniformly superhydrophilic and covered by two-level hierarchical micro-grooves with duckbilled microcavities regularly aligned at groove bottom. Coupling the material property and unique micro hierarchical structures, water in microgrooves can be effectively blocked in backward and continuously climbs forward, and finally creates unidirectional liquid transport with continuous, long-distance, and high-speed superiorities. These natural samples provide significant prototypes for 2D directional liquid transport studies. Apparently, these 2D natural samples' directional liquid transporting ability is strongly related to their micro-nano surface structures and materials properties. The detailed comparison of 1D and 2D natural unidirectional liquid transport are listed in Table 1. Compare to directionally move liquid with external energy, the directional liquid transport on natural samples occurs spontaneously 
Table 1 Typical nature samples with 1D or 2D unidirectional liquid transport

\begin{tabular}{|c|c|c|c|c|c|c|}
\hline Structure type & Nature samples & \multicolumn{2}{|c|}{ Unidirectional transport mechanism } & Contact Angle (CA) & $\begin{array}{l}\text { Transport } \\
\text { speed }\end{array}$ & Ref. \\
\hline \multirow{4}{*}{$1 \mathrm{D}$} & Spider silk & Conical structure & Linear gradient wettability & $<90^{\circ}$ & $\sim 0.148 \mathrm{~mm} \cdot \mathrm{s}^{-1}$ & [19] \\
\hline & Cactus spine & Conical structure & Linear gradient wettability & $113.3^{\circ}-126.5^{\circ}$ & $\sim 0.047 \mathrm{~mm} \cdot \mathrm{s}^{-1}$ & {$[20]$} \\
\hline & Shorebird beak & Wedged structure & & & $\sim 10 \mathrm{~mm} \cdot \mathrm{s}^{-1}$ & {$[17]$} \\
\hline & Sarracenia trichomes & Conical structure & & $\sim 48^{\circ}-65^{\circ}$ & $\sim 11.7 \mathrm{~mm} \cdot \mathrm{s}^{-1}$ & {$[24]$} \\
\hline \multirow{3}{*}{$2 \mathrm{D}$} & Desert beetle's back & \multicolumn{2}{|c|}{ Surface gradient wettability (chemical composition) } & & & {$[25]$} \\
\hline & Butterfly wings & \multicolumn{2}{|c|}{ Surface gradient wettability (anisotropic structure) } & $151.3^{\circ} \pm 0.4^{\circ}$ & & {$[23]$} \\
\hline & Nepenthes alata's peristome & \multicolumn{2}{|c|}{ Gradient Taylor rising \& edge pinning effects } & $\sim 0^{\circ}$ & $\sim 78 \mathrm{~mm} \cdot \mathrm{s}^{-1}$ & {$[15]$} \\
\hline
\end{tabular}
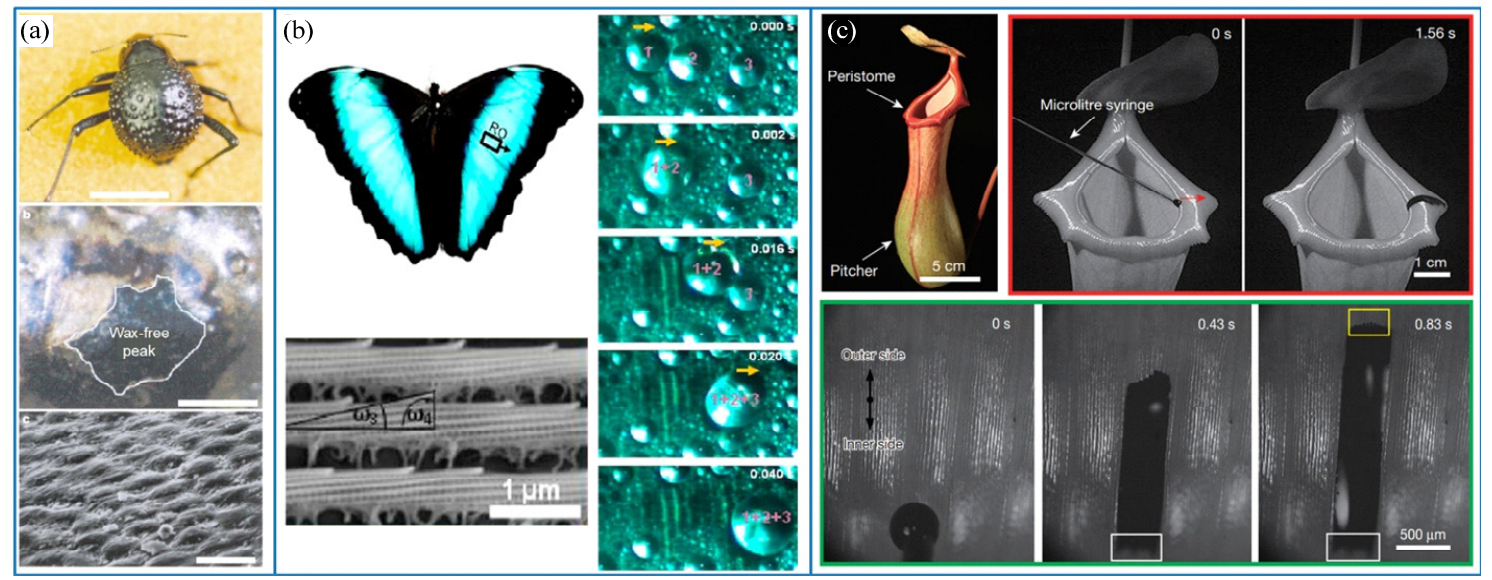

Fig. 2 (a) Desert beetle's (Stenocara sp.) back demonstrates 2D unidirectional liquid transport with liquid aggregating from the hydrophobic area to the hydrophilic area (reproduced from Ref. [15] with permission). (b) Anisotropic structures on butterfly wings (M. deidamia) lead to directional liquid transport during the droplets coalescing (reproduced from Ref. [23] with permission). (c) The peristome of Nepenthes alata exhibits a strong 2D directional liquid transport with high speed and long-distance properties (reproduced from Ref. [25] with permission).

without any energy input, which makes it an ideal prototype for green energy applications ${ }^{[36-38]}$. To effectively apply such natural directional liquid transport, it is critical to reveal the micro-nano liquid-solid interacting effect under 1D and 2D unidirectional liquid transport.

\section{Bioinspired 1D unidirectional liquid transport}

With detailed micro-nano interfacial liquid movement characteristics, the underlying mechanisms on natural samples with 1D directional liquid transport have been revealed. By extracting their feature structure and material properties, various bioinspired 1D unidirectional liquid transport structures have been designed and several fabrication methods have been introduced. Their applications in fog harvesting and oil/water emulsion separation are presented, and the utilization of smart materials on 1D directional transport structures to re- gulate liquid transporting performances are discussed.

\subsection{Mechanisms of 1D unidirectional liquid trans- portation}

The movement of a liquid droplet on the 1D structure is driven by unbalanced forces at opposite sides of a droplet, which can be generated by the geometric gradient or chemical gradient of the material surface. For spider silks and cactus spines, the unbalanced force derives from the Laplace pressure difference induced by their anisotropic 1D conical structure. When droplets flow on the outer surface of a cone spine, the Laplace pressure difference $\Delta P_{\text {Out }}$ can be expressed as:

$$
\Delta P_{\text {Out }}=-\int_{z_{1}}^{z_{2}} \frac{2 \gamma}{\left(r+R_{0}\right)^{2}} \sin \alpha \mathrm{d} z,
$$

where $\alpha$ is the half-apex angle of the cone, $r$ the local mean radius of cone at liquid droplet, $R_{0}$ the drop radius, 
$\gamma$ the surface tension, and $z_{1}$ and $z_{2}$ are the rear and front position of a droplet at the axis of a cone (Fig. 3a) ${ }^{[39]}$. Under such an unbalanced force, the liquid droplet continuously and directionally moves towards the direction of the increasing radius of curvature, i.e. from cone tip to root. Unidirectional liquid transport also occurs in the inner surface of a cone tube. Its Laplace pressure difference $\Delta P_{\text {In }}$ can be described as:

$$
\Delta P_{\mathrm{In}}=4 \gamma\left[\frac{\cos (\theta-\alpha)}{z_{2} \alpha}-\frac{\cos (\theta+\alpha)}{z_{1} \alpha}\right],
$$

where $\theta$ is the contact angle of liquid ${ }^{[40]}$. Its transport direction is based on surface wettability, i.e. liquid droplet transports to the tip side in a hydrophilic tube and to the root side in a hydrophobic tube (Fig. 3b). This directional transport mechanism can also be applied on liquid droplet directional transport between a wedgedshaped corner, such as shorebirds beaks ${ }^{[41]}$, hairs of Chinese brushes ${ }^{[42]}$, or setae of water striders ${ }^{[43]}$.

The gradient wettability is another driving force for liquid droplets. On a gradient wetting surface, liquid droplet forms a smaller contact angle at the more wettable side and a larger contact angle at the less wettable side, which are presented as $\theta_{A}$ and $\theta_{R}$, respectively. The unbalanced wetting force generated at the contact line is:

$$
F=\int_{l_{\text {Rear }}}^{l_{\text {ront }}} \gamma\left(\cos \theta_{A}-\cos \theta_{R}\right) \mathrm{d} l,
$$

where $l_{\text {Front }}$ and $l_{\text {Rear }}$ are the contact line length of the liquid droplet at the front and rear edges, respectively (Fig. 3c $)^{[44,45]}$. Based on Eq. (3), liquid tends to move from a less wettable area to a more wettable area on gradient energy surface. Such gradient wettability can be generated either by gradient surface chemical composition $^{[44,45]}$ or gradient surface roughness ${ }^{[46-48]}$. In particular, the impact of surface roughness is strongly related to liquid contact states. When liquid contact in Wenzel state, the surface roughness's impact on contact angle can be described as:

$$
\cos \theta_{W}=\mu \cos \theta,
$$

where $\mu$ is the surface roughness ratio, $\theta$ and $\theta_{W}$ are the intrinsic contact angles and apparent contact angles ${ }^{[49]}$. For contact in Cassie-Baxter state, the apparent contact angle of Cassie-Baxter state $\theta_{C}$ is:

$$
\cos \theta_{C}=\sigma_{1}(\cos \theta+1)-1
$$

where $\sigma_{1}$ is the fractional surface area of the rough substrate $^{[50]}$. Based on Eqs. (4) and (5), a surface with gradient roughness could create different contact angles at opposite sides of the liquid droplet, where such unbalanced surface tension could directionally move the droplet. This gradient wettability strategy could combine the anisotropic structures to further enhance the directional liquid transport performance, such as spider silks and cactus spines with gradient roughness on their cone's surface.

Different from the gradient roughness on spider silks or cactus spines, the conical shape Sarracenia trichomes are covered with high-low microgrooves, which creates complex liquid flowing states with extremely high directional transporting speed. On a dry trichome, liquid spreading sequentially comes in three states from liquid base front filling, to base following filling, and to top major filling, referred as Mode I (Figs. 4a and 4c, left). The liquid spreading distance in Mode I at time $t$ is determined by:

$$
d_{i}=D_{i} \sqrt{\frac{a \gamma}{\eta}} t
$$

with $i=1-3$, respectively, where $D_{i}$ is the geometrical coefficients of the three different microchannels, $\eta$ the liquid dynamic viscosity, and $a$ the width of the minor base microchannel. After the liquid in Mode I spreads to the trichome root, the trichome surface is left with a thin stable liquid film in low microgrooves, where high microgrooves protrude the liquid film surface and form convex meniscuses. Thus, the solid trichome can be regarded as a liquid cone covered with liquid microgrooves (Figs. $4 \mathrm{~b}$ and $4 \mathrm{c}$, bottom, middle). Such structure and material peculiarities lead to extremely high wettability for liquid droplets and super-slippery for droplet sliding. The following deposited liquid droplets then form an ultrafast directional liquid transporting speed to be about 20 times faster than Mode I and about three orders of magnitude faster than the cactus spine, referred as Mode II (Fig. 4c, right). The liquid transport distance in Mode II at time $t$ can be described as:

$$
d_{3}^{(s)}=D_{3}^{(s)} \sqrt{\frac{a \gamma}{\eta}} t,
$$


(a)

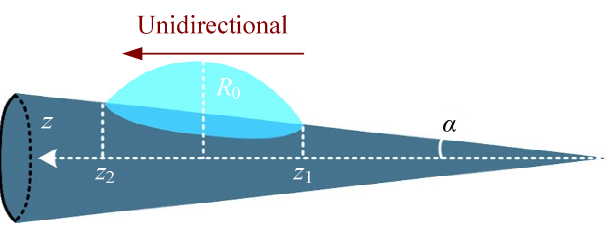

(c)

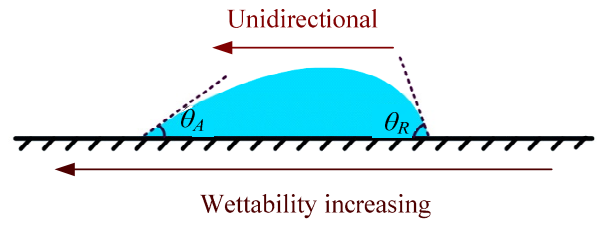

(b)
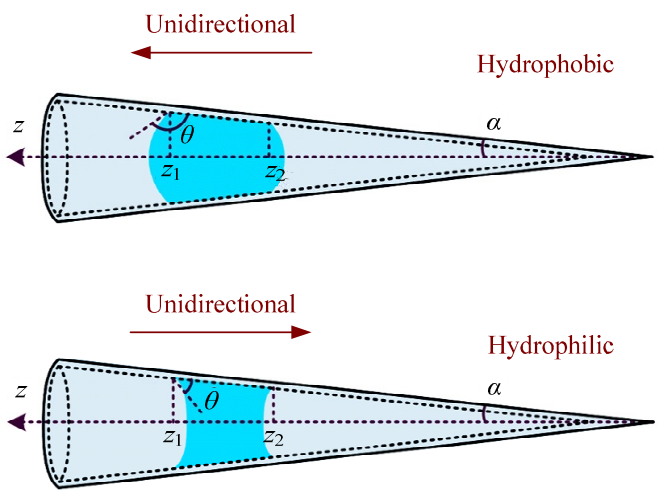

Fig. 3 Mechanisms of 1D unidirectional liquid transport. (a) Liquid directionally transports to the root on the outside surface of a cone with a half-apex angle of $\alpha . R_{0}$ is the diameter of the liquid droplet. $z_{1}$ and $z_{2}$ are the rear and front edges of the droplet in the $z$ direction. (b) Liquid directionally moves to the root in a hydrophobic cone tube and to the tip in a hydrophilic cone tube. $\theta$ is the contact angle. (c) Gradient surface energy induces liquid to directional transport to the wettable side.

(a) Mode-I on dry hierarchical high-low microgrooves

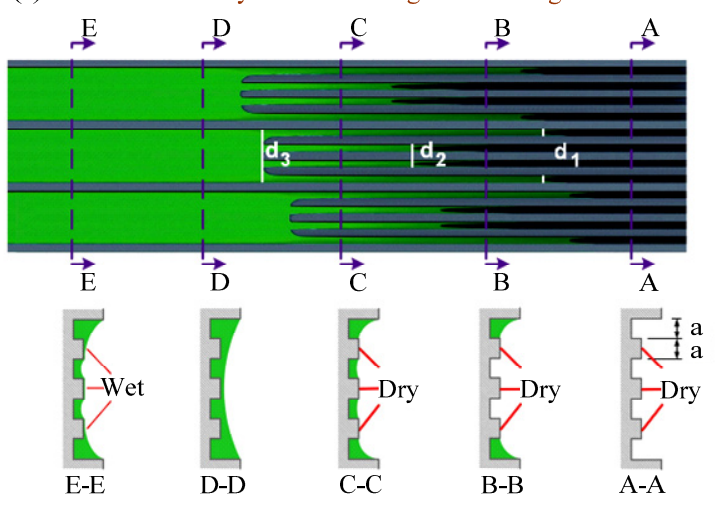

(b) Mode-II on wetted hierarchical high-low microgrooves

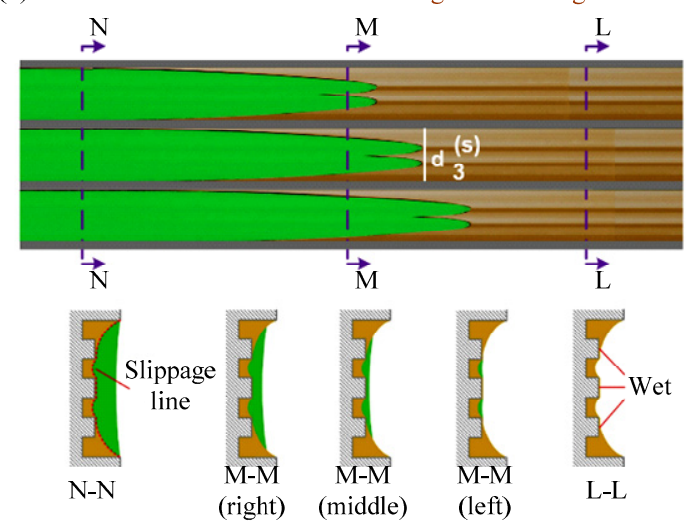

(c)

Mode-I on dry trichome

Mode-II on wet trichome

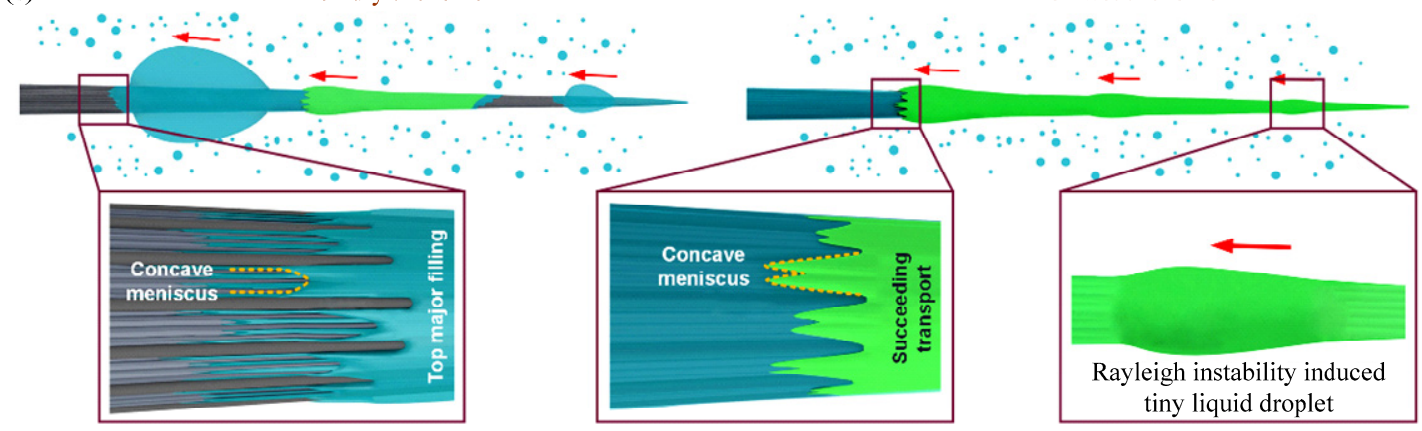

Fig. 4 Mechanisms of ultrafast 1D unidirectional liquid transport. Mode I (a) and Mode II (b) directional liquid transport on dry and wetted hierarchical high-low microgrooves, respectively. (c) Left shows the Mode I that liquid directional transports to root on dry trichome, and a layer of thin liquid film is left in hierarchical microchannels. With the totally wetted surface, an ultrafast liquid unidirectional liquid transport appears, and the liquid transport turns into Mode $\mathrm{II}^{[24]}$.

$$
D_{3}^{(s)}=\sqrt{\frac{2 \varepsilon_{H / h} \cos \theta+n(\cos \theta-1)}{n \varepsilon_{H / h} \beta_{3}^{(s)}}},
$$

where $D_{3}^{(s)}$ is the geometrical coefficient of the major convex meniscus, $\varepsilon_{H / h}=(H-h) / a$ the coefficient of the rib height difference in the microchannel, $\theta$ the contact angle of liquid, and $\beta_{3}{ }^{(s)}$ the dimensionless flow resistance under flow boundary conditions ${ }^{[24]}$.

Based on these theories, several strategies could be used to adjust the 1D liquid directional transporting 
performances, i.e. the anisotropy strength and the directional transporting speed. On the 1D conical structure, higher surface wettability and larger cone apex angle could provide stronger Laplace pressure, thus, enhance the liquid directional transporting speed. Such performance could be further increased with gradient surface wettability from either gradient chemical composition or gradient surface roughness. On Sarracenia trichomes, the ultrafast liquid directional transport resulted from the high-low microgrooves could be affected by the height of their sidewalls, where higher sidewalls for low- microgrooves and larger height differences between highlow microgrooves both could lead to faster liquid transporting speed. With these principles, the design of 1D unidirectional liquid transporting structures can be optimized and the adjusting of liquid transporting performance could be realized.

\subsection{Bioinspired design and fabrication of 1D unidi- rectional liquid transportation}

Based on the natural samples' characterization and mechanisms revealing, conical or wedged-shaped structures and surface gradient wettability are extracted to be the two key factors for 1D unidirectional liquid transportation. Various spindle knots wires or long sharp cones are designed to mimic the shape of spider silks or cactus spines. Several gradient wettability modification methods have been introduced to cooperate with the feature structures to further enhance the 1D directional transport effect. In this section, these fabrication methods have been listed and their advantages and disadvantages have been compared.

\subsubsection{Spindle knots structure fabrication}

As one main type of 1D unidirectional liquid transport structure, spider silk inspired spindle knots wire can be fabricated by various methods, including dip-coating $^{[26,51]}$, microfluidics ${ }^{[52-54]}$ and chemical gradient erosion ${ }^{[55]}$. By drawing out fibers from solution with coating materials, a thin layer of solution is deposited on it and then spontaneously breaks into numerous droplets with uniform gap distance under Rayleigh instability (Fig. 5a) ${ }^{[26]}$. After the evaporation of the solvent, coating materials in droplets dissolve out and form equidistance spindle knots on the fiber with a shape like spider silks, which exhibits a remarkable 1D unidirectional liquid transporting ability. By choosing different coating materials, such as poly(methyl methacrylate) (PMMA), poly(vinyl acetate) (PVAc), polystyrene (PS) and poly(vinylidene fluoride) (PVDF), the surface energy of spindle-knots can be adjusted to control the liquid transporting speed on the wire ${ }^{[52-54]}$. To in-situ control the structure size of spindle knots wire, a microfluidic technique integrating three coaxial fluids is introduced with processes including microfiber joint spinning, fluid coating, and knot emulsifying, which can continuously produce spindle knots wire (Fig. 5b). By controlling the flow rate of these three fluids, the size and spacing of spindle-knots can be precisely adjusted to control the liquid directional transporting ability ${ }^{[53]}$. Due to the short gap distance between spindle knots on spider silks, its directional liquid transporting distance is limited to half-length of spindle knots. Therefore, a gradient spindle knots fiber is promoted by tilt dip-coating methods (Fig. 5c) to stronger directional transporting ability, where the deposited water drops can be continuously driven from small knots to large knots with distance in millimeter-scale, much longer than spider silk's micrometer scale ${ }^{[55]}$.

\subsubsection{Conical structure fabrication}

As another dominant 1D unidirectional liquid transport structure, the cactus spine inspired cone structure has a length of several centimeters, which is much longer than spindle knots of spider silks and could promote much longer directional and constant liquid transport distance. A single long cone can be easily achieved by a gradient chemical etching method, where a metal wire or polymer fiber is drawn out from corrosive acid or organic solvent at a constant speed (Fig. 6a) ${ }^{[39]}$. By controlling the electric current in the electrochemical etching process, a designed integrating tapered wire can be achieved with continuous directional water transporting ability ${ }^{[56]}$. A star-shaped hydrophilic pattern can be fabricated with patterned UV exposure, where its sharp wedged edges can directionally aggregate fog more efficiently than round edges ${ }^{[57]}$. Tree-shaped hydrophilic with hierarchical wedged edges can be fabricated by laser writing method, which demonstrates continuous directional transporting ability 


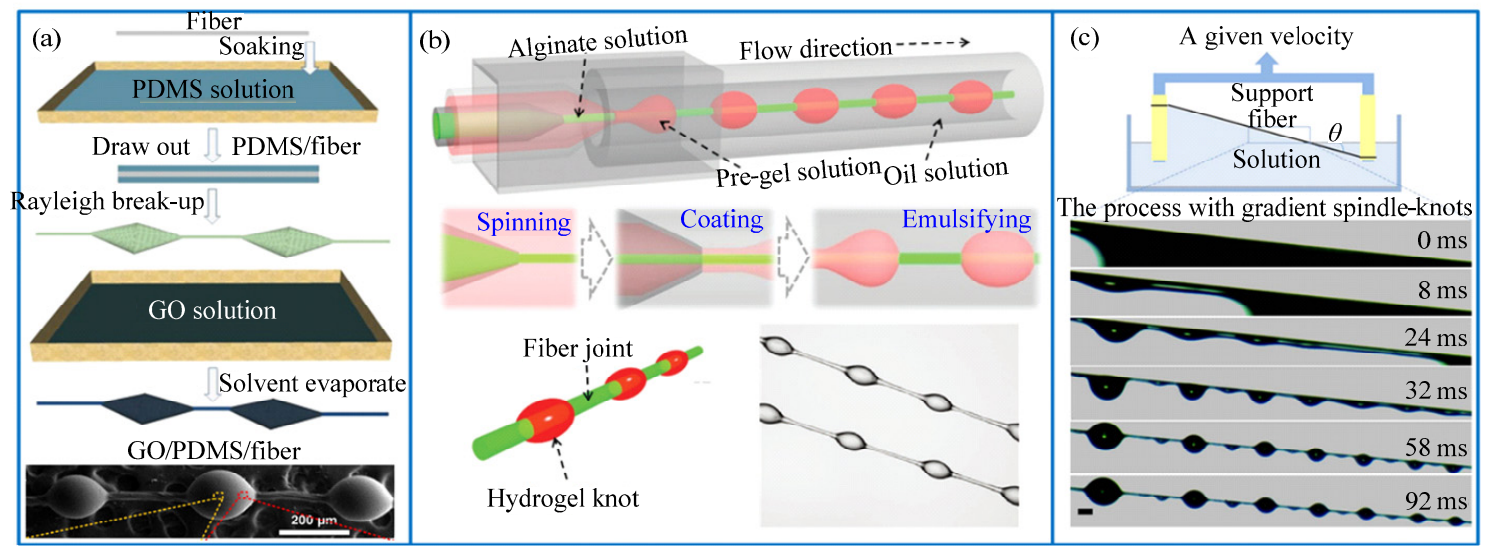

Fig. 5 (a) Fabrication of spindle knots wires with the dip-coating method. After coating a wire with a layer of PDMS liquid film, the film spontaneously turns into uniformly spaced spindle knots under the Rayleigh instability effect (reproduced from Ref. [26] with permission). (b) Continuous fabrication of spindle knots wires with microfluidics techniques (reproduced from Ref. [53] with permission). (c) To achieve longer directional liquid transport, gradient sized spindle knots are introduced with the tilted dip-coating method (reproduced from Ref. [55] with permission).

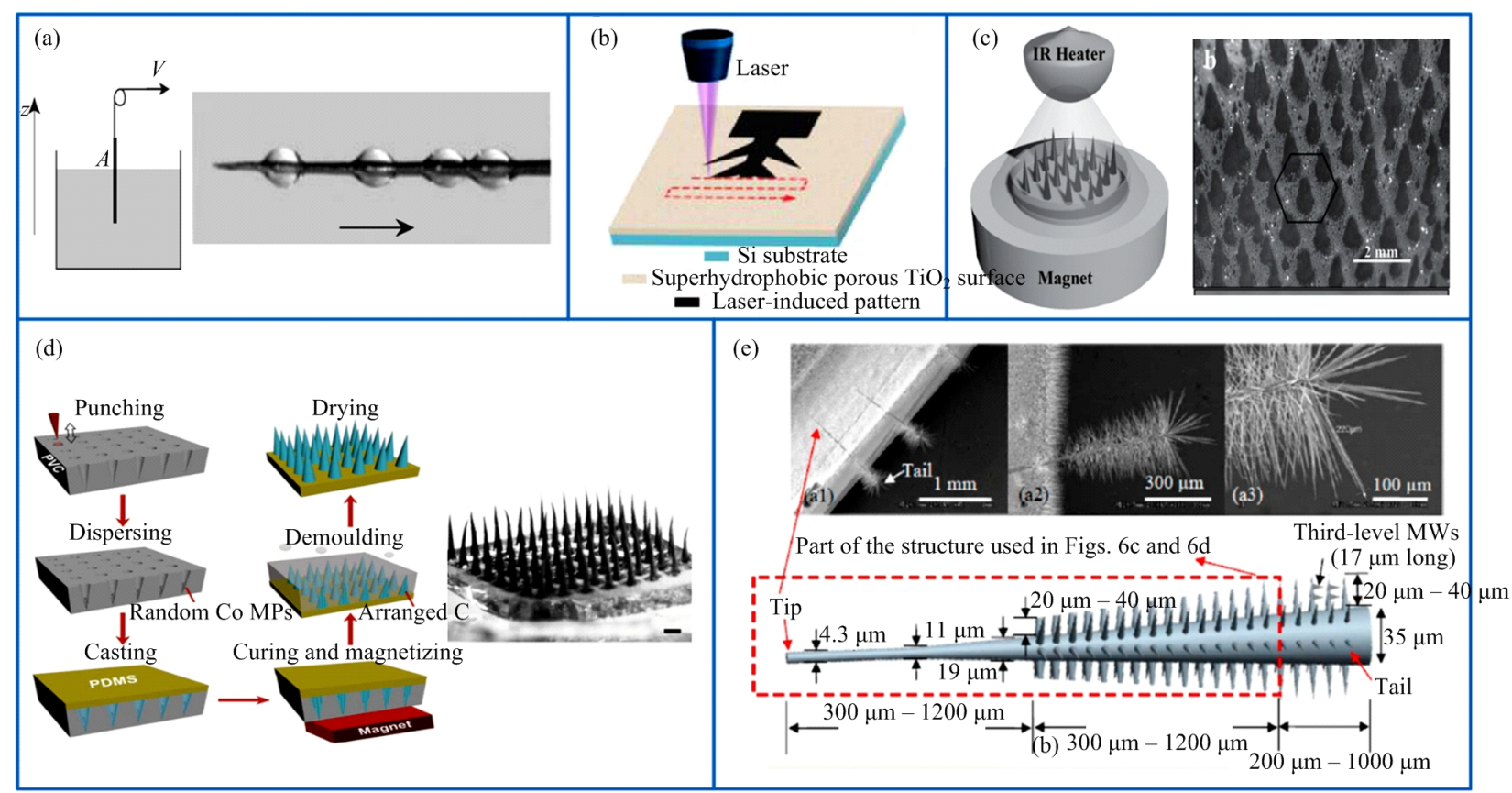

Fig. 6 (a) A long cone wire can be achieved by gradient etching method with a wire gradually pulled out from the etching solution (reproduced from Ref. [39] with permission). (b) Hydrophilic hierarchical wedged pattern with superhydrophobic periphery fabricated by laser writing method (reproduced from Ref. [58] with permission). (c) Utilizing the unique topography of ferrofluid under the magnetic field, cone array can be rapidly fabricated with uniform height and spacing (reproduced from Ref. [59] with permission). (d) Cone array replicates from conical cavities fabricated by sharp needle punching (reproduced from Ref. [27] with permission). (e) A hierarchical micro-nano cone structure is fabricated by the Vapor-solid method (reproduced from Ref. [60] with permission).

with liquid moving from small to large wedges (Fig. 6b) ${ }^{[58]}$. To enhance the $1 \mathrm{D}$ directional liquid transport performance, surfaces with cone array are introduced to simultaneously transport liquid droplets on multiply cones. Using prepolymer (e.g. polydimethyl- siloxane (PDMS)) as a carrier fluid, a uniform cone array surface can be attained by solidifying ferrofluid under a static magnetic field, which is more suitable for large-area production (Fig. 6c) ${ }^{[59]}$. The cone array can also be achieved by easy polymer replication with a cone 
cavities mold, which can be fabricated by mechanical processes such as needle stabbing or laser punching on substrate (Fig. 6d) ${ }^{[27]}$. Inspired by the hierarchical cone structure from cactus spine, branched $\mathrm{ZnO}$ nanowire with hierarchical micro-nano cone is introduced by Vapor-solid deposition method to successively grow first-level stem cone and second-level nano branched cones. It shows even stronger 1D unidirectional liquid transporting ability than cactus spine (Fig. 6e) ${ }^{[60]}$.

\subsubsection{Integration of surface gradient wettability and} conical structure

The surface gradient wettability is another driving force for 1D unidirectional liquid transport. However, it induced driving force is relatively weak compared to the Laplace pressure difference on conical structures that the motion of droplet can be easily blocked by surface defects due to the contact line hysteresis effect. Thus, the gradient surface wettability usually works as an auxiliary for 1D conical structures to enhance directional liquid transporting ability ${ }^{[61]}$, which can be created by gradient surface roughness or gradient chemical composition. The gradient roughness can be self-formed by the dip-coating method during the gradient volatilization of solvents on spindle knots wire ${ }^{[26,52,54]}$. The gradient chemical composition can be achieved by gradually withdraw a wire from the surface grafting solution (Fig. 7a) ${ }^{[28]}$. The gradient chemical modification can also be achieved by graphite oxidation, where a gradient electric field forms between a large graphite cathode and a small copper anode (Fig. 7b) ${ }^{[62]}$.

With these fabrication methods, the spider silk and cactus spine inspired 1D liquid directional transporting structures could be achieved and their directional transporting performances vary largely. Since the conical structure on the spindle knot wire is short, its liquid directional transporting distance is limited to about several hundred micrometers and the speed is relatively slow to about hundreds of millimeters per minute. On the gradient spindle knot wire, liquid droplets could form another level of directional transport between the gradient adjacent knots, thus the directional transporting distance and speed could be largely enhanced to several millimeters and hundreds of millimeters per second, respectively. Compare to the knots formed single wire structure, the cones are more easily to be designed and fabricated into arrays, which helps form directional liquid transport with a higher transport volume rate.

\section{Bioinspired 2D unidirectional liquid transport}

This section summarizes the underlying mechanisms of 2D unidirectional liquid transport on surfaces under the cooperative effect of featured 2D micro-nano structures and surface material properties. Based on these mechanisms, various bioinspired 2D directional liquid transport surfaces have been designed and fabricated. Their applications in microfluidics, biomedical devices, and continuous oil/water separation have been presented. With the emergence of all kinds of smart materials, the regulations of 2D liquid transport in directional guiding, transporting speed, and liquid merging are discussed.

\subsection{Mechanisms of 2D unidirectional liquid trans- portation}

2D unidirectional liquid transport can also be triggered by gradient surface wettability, similar to the directional liquid transport in $1 \mathrm{D}$ as presented in section 3.1 and Fig. 3c. Due to the different surface energy on hydrophilic and hydrophobic areas, liquid tends to expand on the hydrophilic area and retract on the hydrophobic area, eventually forms the directional liquid transport. Such directional liquid transport can hardly create long-distance transport as the gradient of wettability decreases with the increase of distance. Fortunately, on butterfly wings, the periodically inclined cuticle lamella formed asymmetric array structures could generate a much longer directional liquid transport distance. Inspired by the butterfly wings' asymmetric structures, inclined pillar arrays surface has been introduced to create long-distance 2D directional liquid transport, where liquid spreads to the pillar inclined side and stops at the opposite side. The spreading of liquid results from that the liquid contact line continuously reaches adjacent pillars to form successive spreading, and this only happens when liquid intrinsic contact angle $\theta_{0}$ is smaller than the critical angle $\theta_{c r}=\tan ^{-1}\left(H_{\text {eff }} / l_{\text {eff }}\right)$, where $H_{\text {eff }}$ and $l_{\text {eff }}$ are the effective height and length of pillars, respectively (Fig. 8a). For inclined pillar arrays 


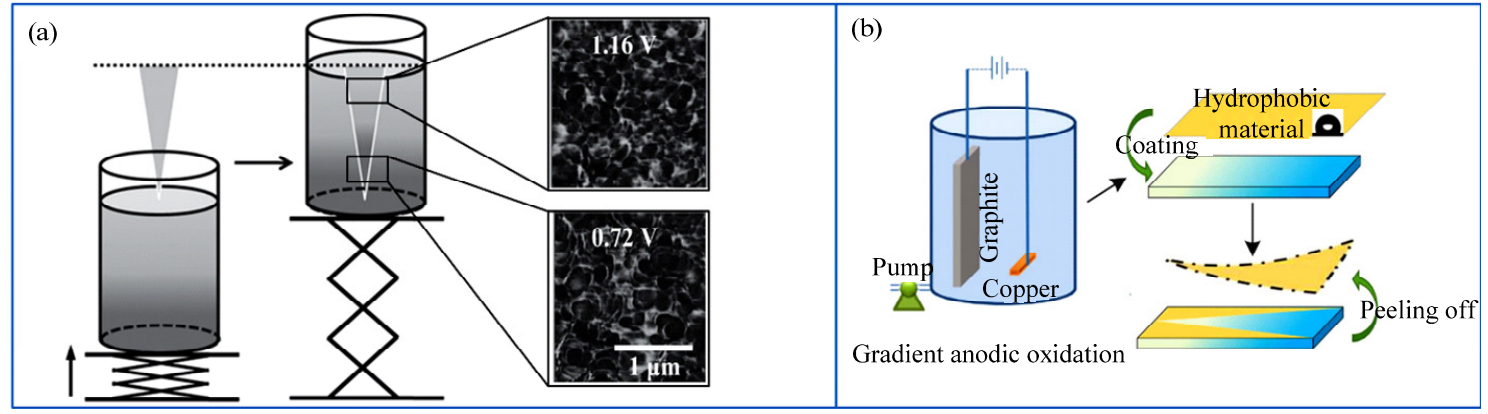

Fig. 7 (a) Surface gradient chemical modification by gradually withdrawing cone from surface grafting solution (reproduced from Ref. [28] with permission). (b) Surface chemical modification with graphite oxidation. The gradient wettability is formed by the varied distance from anode and cathode (reproduced from Ref. [62] with permission).

(a)
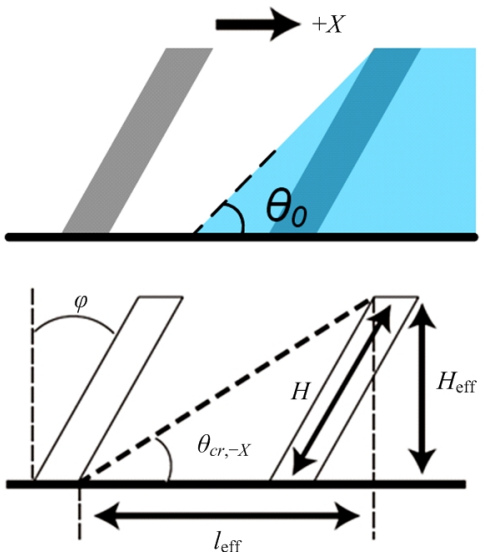

(b)

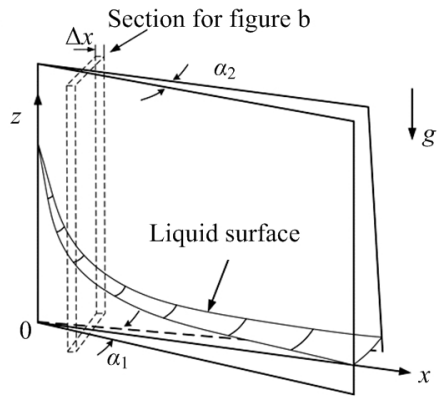

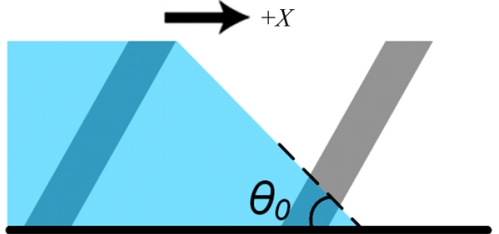
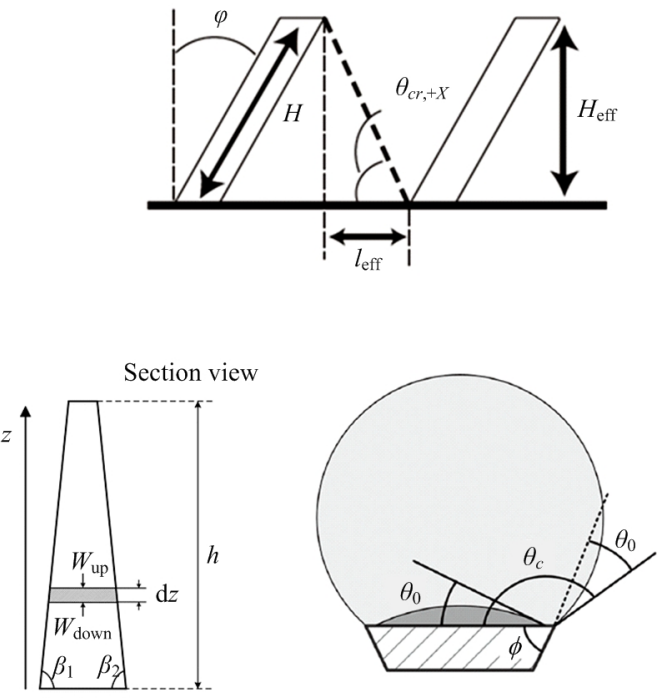

Fig. 8 (a) The mechanism of 2D unidirectional liquid transport induced by an inclined pillar array. The capillary attraction in pillar inclined direction is higher than in the opposite direction and leads to the directional transport of liquid (reproduced from Ref. [63] with permission). (b) On Nepenthes peristome surface, the liquid is dragging forward by gradient capillary rise effect from the duckbilled cavities and pinned backward by edge pinning effect (reproduced from Ref. [25] with permission).

surface, two critical angles can be defined as in pillar inclined direction $\theta_{c r,+X}$ and the opposite direction $\theta_{c r,-X}$, which can be presented as:

$$
\left\{\begin{array}{l}
\theta_{c r,+X}=\tan ^{-1} \frac{(H / L) \cos \varphi}{1-(H / l) \sin \varphi} \\
\theta_{c r,-X}=\tan ^{-1} \frac{(H / L) \cos \varphi}{1+(H / l) \sin \varphi}
\end{array},\right.
$$

where $H, l$, and $\varphi$ are pillar height, pillar spacing, and inclined angle $\varphi$, respectively ${ }^{[63]}$. Only with $\theta_{c r,-X}<\theta_{0}<\theta_{c r,+X}$ can the liquid be dragged forward to pillar inclined direction and pinned in opposite direction, which eventually forms 2D unidirectional liquid transporting.

The 2D unidirectional liquid transport on $\mathrm{Ne}$ penthes peristome is composed of two liquid adjusting 
effects, i.e. liquid capillary rising in the forward direction and edge pinning in the backward direction. In the forward direction, the hydrophilic duckbilled arch and microgroove bottom form a microcavity with a sharp wedge corner angle to generate the capillary dragging force. This liquid dragging force can be quantified as the capillary rising height at the intersecting corner of two vertical placed plates with intersecting corner angle $\alpha$. For two plates with constant $\alpha$, the capillary rise height at position $x, H_{e}(x)$, can be described as:

$$
H_{e}(x)=\frac{2 \gamma \cos \theta}{\rho g x \alpha}
$$

where $\gamma, \rho, \theta$, and $g$ represent the surface tension, the liquid density, the liquid contact angle, and the gravitational constant, respectively ${ }^{[64,65]}$. However, the microcavities on Nepenthes evolve further beyond this model to a gradient wedge angle, which can be presented as a gradient capillary rise with bottom $\alpha_{1}$ shrinking to top $\alpha_{2}$ (Fig. 8b, left). The gradient capillary height $H_{e}(x)$ can be presented as:

$$
H_{e}(x)=\frac{2 \gamma \cos \theta}{\rho g x \alpha_{1}}+\frac{\alpha_{1}-\alpha_{2}}{\alpha_{1} h} \frac{4 \gamma^{2} \cos ^{2} \theta}{\rho^{2} g^{2} x^{2} \alpha_{1}^{2}},
$$

where $h$ is the height of the intersecting plates ${ }^{[25]}$. Since the surface is superhydrophilic with $\theta$ close to $0^{\circ}$ and $\alpha_{1}>\alpha_{2},\left.H_{e}(x)\right|_{\alpha_{1}>\alpha_{2}}$ is larger than $\left.H_{e}(x)\right|_{\alpha_{1}=\alpha_{2}}$, which indicates these microcavities with shrinking corner on Nepenthes peristome can generate stronger capillarity than that with a uniform corner, to drag liquid transport forward with higher speed.

In the backward direction of Nepenthes peristome, the blocking of liquid is another critical factor for unidirectional liquid transport. In each second-level microgroove, the duckbilled arch of microcavity owns a sharp edge with a sectional angle of $\sim 5^{\circ}$. This sharp edge can form the Gibbs inequality condition (Fig. 8b, right), and the equilibrium contact angle $\theta$ at edge remains in a range of

$$
\theta_{0} \leq \theta \leq \theta_{C},
$$

where $\theta_{0}$ is the intrinsic contact angle of the surface, $\theta_{C}$ the critical contact angle when liquid spreads spontaneously down the edge ${ }^{[66]}$. $\theta_{C}$ is defined by the edge sectional angle $\phi$, where $\theta_{C}=180^{\circ}-\phi+\theta_{0}$. When liquid flows backward and meets the duckbilled arch edge, this sharp edge creates a large critical contact angle $\theta_{C}$ to effectively block liquid flowing backward even the material is superhydrophilic. Combining the strong liquid capillary effect in the forward direction and sharp edge pinning effect in backward, the unidirectional liquid transport on 2D surfaces is generated on Nepenthes peristome with continuous, long-distance, and high-speed peculiarities.

With these mechanisms, the 2D directional transporting performance can be adjusted by tuning the feature structures or surface material wettability. Basically, to let the liquid spreading on a surface, the hydrophilicity of the surface is always necessary. On inclined pillar arrays surface, a higher pillar inclined angle can increase the forward spreading speed and backward pinning ability. Denser pillar array could also increase the forward spreading speed but may fail the backward pinning and result in bidirectional spreading. For Nepenthes peristome mimetic surface, smaller channel width and sharper duckbilled arch can form higher liquid capillarity in the forward direction, which could lead to faster directional liquid transport. These liquid adjusting strategies could give clearer design principles for 2D unidirectional liquid transport.

\subsection{Bioinspired design and fabrication of 2D unidi- rectional liquid transportation}

Based on the origin of liquid driving and pinning forces, 2D directional liquid transport from natural sample surfaces can be classified into three types, including patterned wettable surfaces, inclined pillar arrays surfaces, and Nepenthes peristome mimetic surfaces. Based on these nature samples, various bioinspired 2D directional liquid transport surfaces have been designed, and many micro-nano manufacturing processes have been developed for their application. By adjusting the material properties or featured structures, their influences on unidirectional liquid transporting performance have been thoroughly compared and their underlying mechanisms have been validated.

\subsubsection{Gradient and patterned wettability surfaces}

Under the inspiration of desert beetle's back, 2D directional liquid transport surfaces with gradient 
chemical composition can be fabricated with similar methods as described on 1D structures in section 3.2.3. By specially designing the gradient wettability on surfaces, the regulations of liquid transport can be realized, such as unidirectional transporting droplets or merging two droplets (Fig. 9a) ${ }^{[67]}$. Garrod et al. reports a two-step plasmachemical methodology and fabricated a patterned superhydrophobic-superhydrophilic with fog condensing ability ${ }^{[68]}$. Zhai et al. have fabricated a patterned wettability surface by micropipetting hydrophilic solution droplets onto a superhydrophobic substrate ${ }^{[69]}$. Apart from gradient surface chemical composition, surfaces with gradually deformed microstructures can also create gradient wettability for 2D directional liquid transport, e.g. micro-channels with gradually increased channel width (Fig. 9b) ${ }^{[70]}$, radially distributed microstrips with gradually enlarged stripe spacing (Fig. 9c) ${ }^{[71]}$, or radially distributed micro pillars ${ }^{[72]}$.

By configuring periodical micropatterns onto a surface, patterned wettability can be achieved to realize a more complex control of 2D liquid flowing. As shown in Fig. 9d, a directional liquid transport surface is created with an asymmetric 2D water barrier pattern, achieved by computerized drawing hydrophobic oil ink lines on a hydrophilic substrate. The pattern is designed with a channel formed by two parallel lines to restrict the flow of liquid, and periodic wedged corners formed by shorter lines to make the liquid flow directional dependently ${ }^{[73]}$. Due to the different contact line lengths in opposite directions formed by the hydrophobic wedged corners, asymmetric liquid flowing resistance is created to form directional liquid flow. Based on this method, various patterns are carried out for liquid transport regulation, such as liquid sequentially flowing at an intersection by drawing wedged corners with different angles, or liquid directionally flowing in a circular or zigzag-shaped pattern. Similar to periodically arranged wedged corners, arrow-shaped pillar arrays can also be used to regulate liquid transport (Fig. 9e). With different arrowheads position and direction, the liquid can be controlled to transport in the forward direction or lateral direction ${ }^{[74]}$. Since such patterned wettability has a periodical feature, its directional liquid transport performance is homogenous and can be achieved on any part of the surface, which makes it is more suitable for large-area applica- tions than gradient wettability surface.

\subsubsection{Inclined pillar arrays surfaces}

Based on the mechanism of inclined pillar arrays induced liquid directional transport, several designing and fabricating methods have been introduced for 2D unidirectional liquid transport. As shown in Fig. 10a, the nanopillar arrays surface is firstly fabricated by project lithography and high aspect ratio etching. Then, the inclination of nanopillars is formed by golden film sputtering with different tilting angles ${ }^{[63]}$. Another more convenient method to achieve inclined pillar arrays surface is to replicate pillar arrays from a cavity arrays mold and bend these pillars under external pressure to form uniform inclination ${ }^{[75]}$. By replicating with temperature response and shape memory material, the pillar inclination can be adjusted under different times of reheating process (Fig. 10b). The ferrofluid can also create inclined pillar arrays surface by placing under a tilted magnetic field. Using pre-polymer as the substance of ferrofluid, the inclined pillar arrays can be solidified and fixed ${ }^{[76]}$, and the pillars inclined angle is tuned by changing the tilted angle of the magnetic field (Fig. 10c). This method is very suitable for rapidly and large-area fabrication of controllable inclined pillars surface.

On these inclined pillar arrays surfaces, liquid directional transporting performance is strongly affected by the structural features of pillars, including material contact angle, pillar inclined angle, and pillars density. With a larger pillar inclined angle, the liquid directional transporting ability on bioinspired surfaces increases. Smaller contact angle or higher pillars density also leads to higher transporting ability, but they also may result in bi-directional transport based on Eq. (9). Thus, the directional liquid transport on inclined pillar surfaces is always limited to a relatively slow speed of $\sim 1 \mathrm{~mm} \cdot \mathrm{s}^{-1}$.

\subsubsection{Nepenthes peristome inspired surfaces}

Nepenthes peristome inspired surfaces have attracted wide attention since its transport speed and distance are much higher than the patterned wettable surface and inclined pillar arrays surface. The easiest way to achieve a Nepenthes peristome mimetic surface is by replicating from the natural Nepenthes peristome samples. After modifying the mimetic surface into 


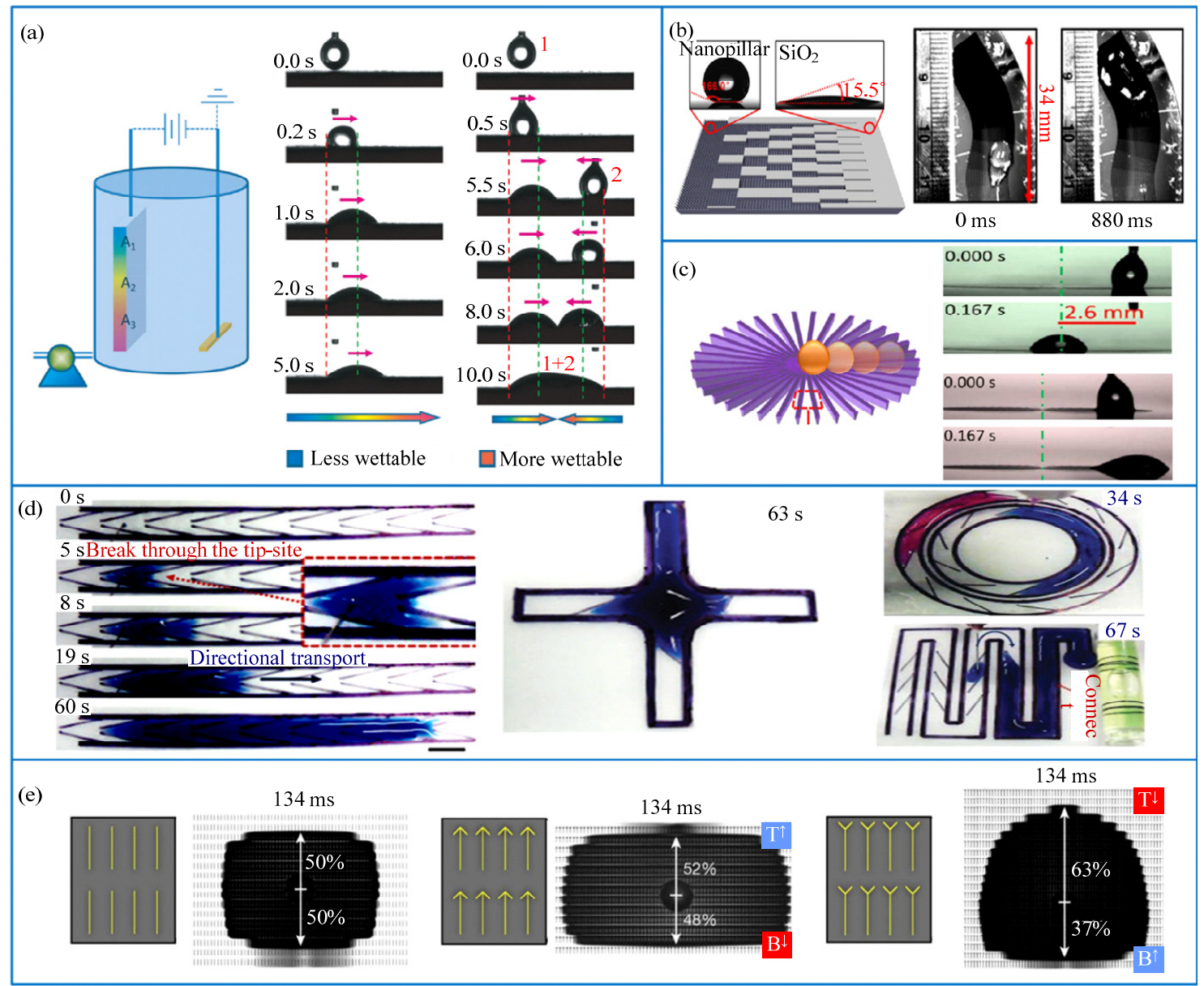

Fig. 9 Liquid directional transported by surface gradient wettability, which can be formed by either (a) gradient chemical composition (reproduced from Ref. [67] with permission) or (b, c) gradient surface structures (reproduced from Refs. [70, 71] with permission). Liquid directional transported by patterned surface wettability, which is formed by (d) hydrophobic/hydrophilic pattern (reproduced from Ref. [73] with permission) and (e) anisotropic structure pattern (reproduced from Ref. [74] with permission).

superhydrophilic, 2D unidirectional liquid transport can be achieved on it just as excellent as on Nepenthes peristome $^{[29,77]}$. Through microscale characterization of Nepenthes peristome, the anisotropic structural features have been extracted to be microgrooves with microcavities constructed by duckbilled arches at groove bottom. To rapidly and large-area fabricate the microgrooves with microcavities, mold replication is a useful method and many fabrication processes have been carried out $^{[78,79]}$. By building the peristome 3D model, bioinspired unidirectional liquid transport surface is replicated from a high-resolution stereo lithography fabricated mold (Fig. 11a) ${ }^{[80]}$. Different liquids including water, ethanol and silicon oils, etc. have been tested on this surface, and it shows that only wettable liquid can be unidirectionally transported ${ }^{[81,82]}$. Another mold replica- tion is based on the micro-cones array formed by ferrofluid self-assembly ${ }^{[33]}$. By tuning the strength and direction of the magnetic field, the microcavities' inclination, length and shape can be controlled to achieve different inclined pillar arrays surfaces (Fig. 11b). Since the width of these microgrooves (about hundreds of micrometers) are much larger than the natural sample's $\sim 50 \mu \mathrm{m}$, its capillarity for liquid forward driving is restricted, and the liquid transport speed on the bioinspired surfaces $\sim 1 \mathrm{~mm} \cdot \mathrm{s}^{-1}$ is much slower than nature sample's $\sim 78 \mathrm{~mm} \cdot \mathrm{s}^{-1}$.

Utilizing semiconductor manufacturing processes, Nepenthes peristome mimetic surfaces can be fabricated with featured structure sizes much closer to natural sample, as shown in Fig. 11c. The surface exhibits a strong unidirectional transporting ability with speed as 
(a)
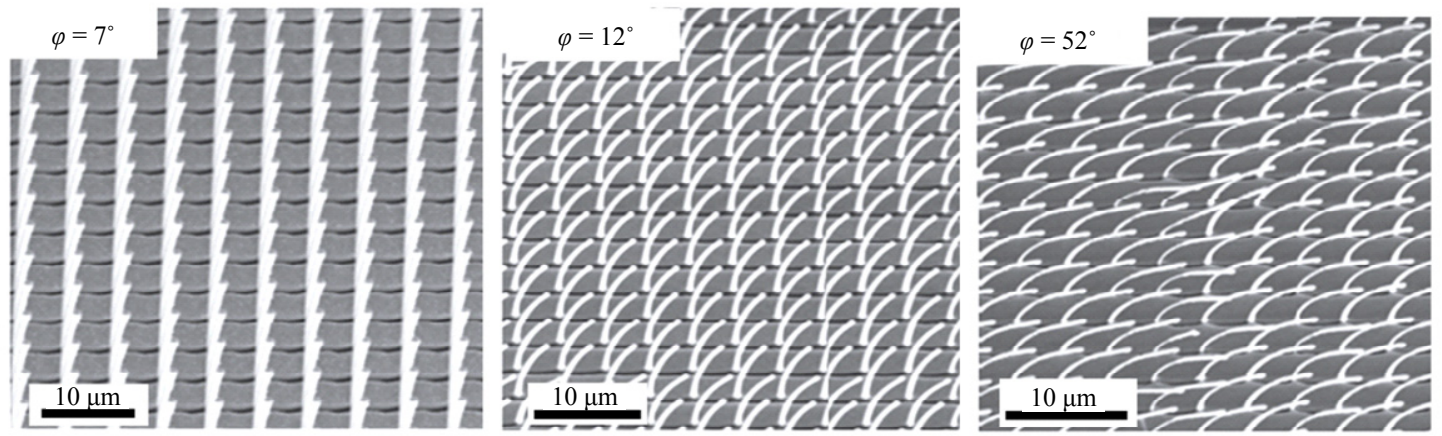

(b)
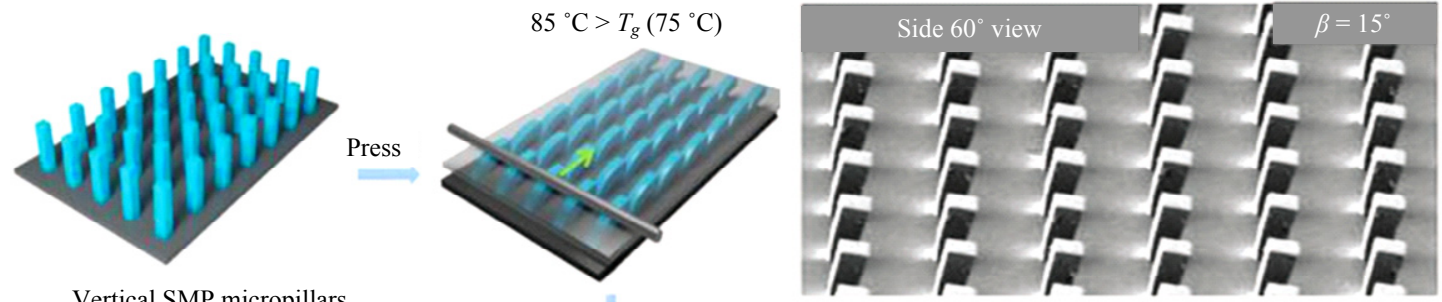

Vertical SMP micropillars

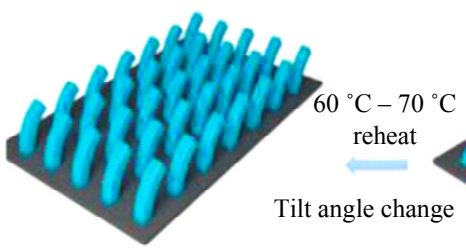

Tilt angle SMP micropillars

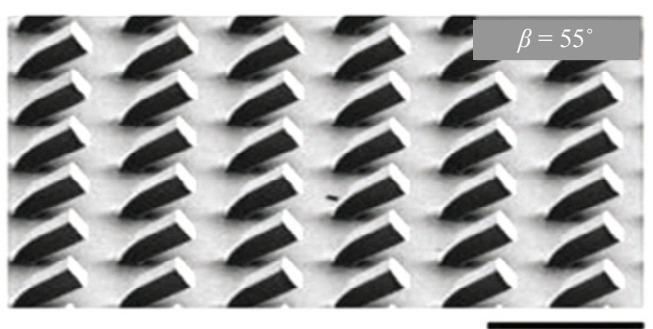

(iii)

(c) (ii)

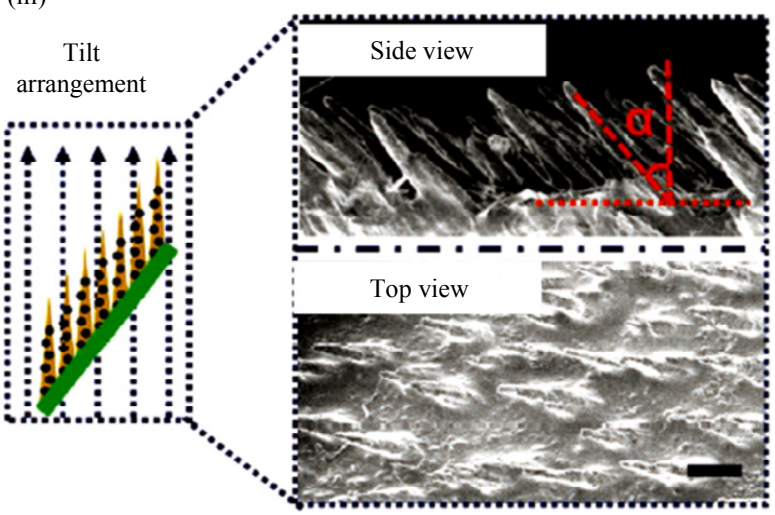

Fig. 10 (a) Nanopillar arrays fabricated by silicon semiconductor processes. The pillars are uniformly bent by the tilted gold sputtering process (reproduced from Ref. [63] with permission). (b) Pillar arrays surface is replicated from cavity arrays mold by temperature response shape memory material. The pillars are firstly bent by external pressure, then the inclination can be adjusted by controlling the time of reheating processing (reproduced from Ref. [75] with permission). (c) The uniform pillar array is self-formed with ferrofluid under a magnetic field. The pillar inclination can be adjusted by changing the direction of the magnetic field (reproduced from Ref. [29] with permission).

fast as $\sim 10 \mathrm{~mm} \cdot \mathrm{s}^{-1}$ and can be easily fabricated into circular or spiral-shaped patterns to guide liquid directional transport ${ }^{[83]}$. Another more convenient fabricating method is introduced with two steps inclined photolithography of SU-8 photoresist, i.e. tilted exposure to build inclined arc pits array, and second exposure to build microgrooves to preciously cover these pits (Fig. 11d) ${ }^{[30]}$. Liquid flowing characterization shows that both the pit edge curvature and pit inclined angle could affect liquid spreading in the forward direction and 

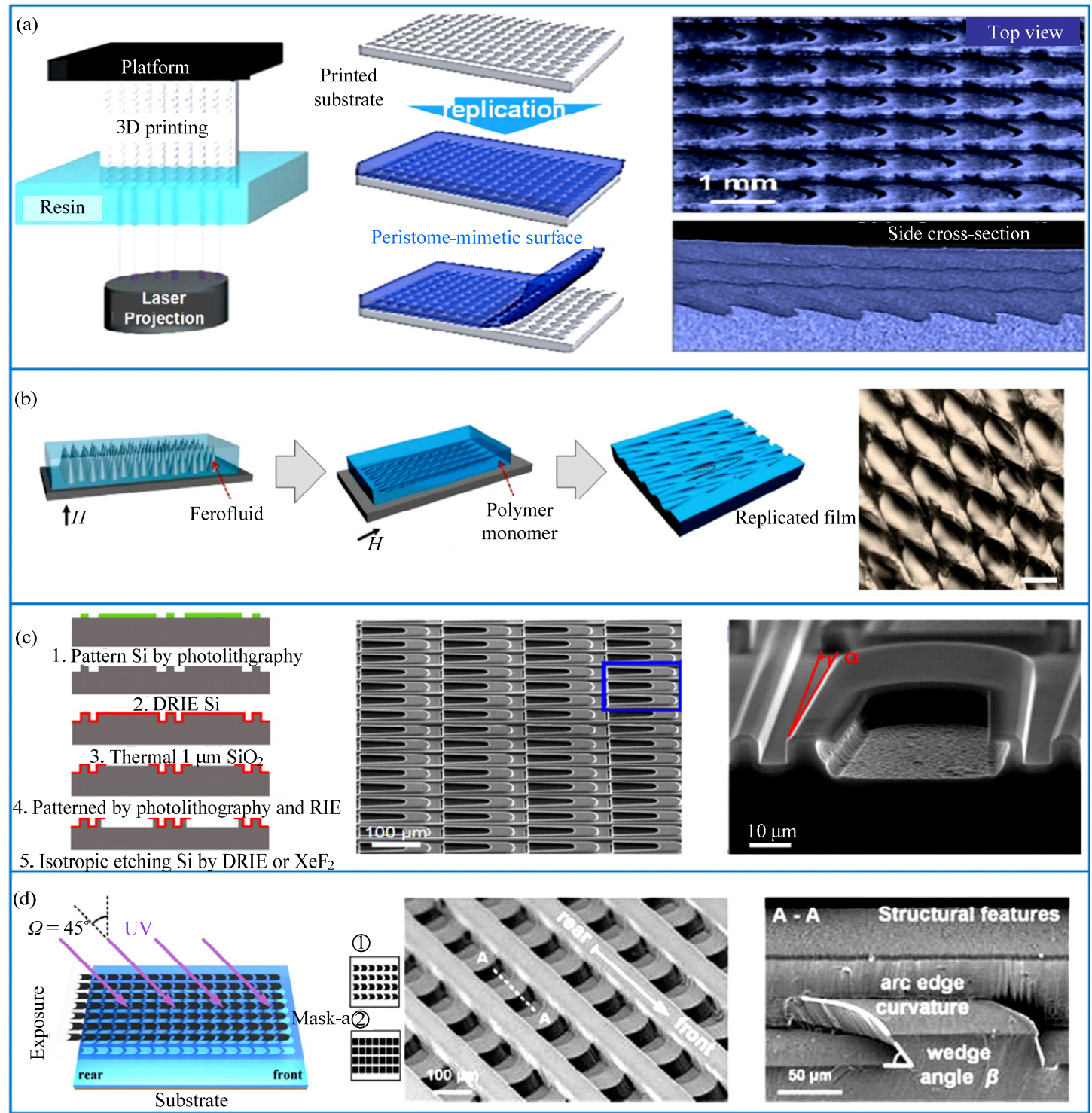

Fig. 11 (a) Nepenthes peristome mimetic surface achieved by replication with a natural Nepenthes peristome sample (reproduced from Ref. [80] with permission) or a 3D printed mold (reproduced from Ref. [82] with permission). (b) Nepenthes peristome mimetic surface via replication of an inclined pillar arrays surface formed by ferrofluid (reproduced from Ref. [33] with permission). (c, d) More exquisite Nepenthes peristome mimetic surfaces can be fabricated by silicon semiconductor processes or SU-8 photography (reproduced from Refs. $[30,83,84]$ with permission).

pinning in the backward direction. By increasing the edge curvature or decreasing pit inclination, the flow of liquid can be tuned from anisotropic spreading to completely unidirectional transport, which provides a structure-based controlled strategy for 2D liquid directional transport ${ }^{[84]}$. Owing to the strong capillarity of smaller microgrooves, this bioinspired surface generates a much higher liquid transport speed of $\sim 12 \mathrm{~mm} \cdot \mathrm{s}^{-1}$. These bioinspired fabrication methods become the fun- damental of 2D unidirectional liquid transport applications in various fields.

As listed above, three types of surfaces are designed and fabricated with patterned surface wettability or unique featured structures to perform the $2 \mathrm{D}$ directional liquid transporting ability. The patterned wettability surface could be obtained by UV exposure with a patterned mask or drawing oil lines on hydrophilic substrates, which are low-cost and suitable for large-area 
fabrication. However, due to their low forward liquid driving force, their directional transporting speed is relatively slow and distance is short. Besides, their forward driving force declines with the pattern size decreases, so that the micro-miniaturization of such patterned wettability surface could result in liquid being unable to transport. On the contrary, smaller structure sizes could lead to better transporting ability on the inclined pillar array surface and Nepenthes peristome mimetic surface, because their driving force is from the featured structures' capillarity. The fabrication of the inclined pillar array surface is easier than the Nepenthes peristome mimetic surface, whose featured structures are hierarchical microgrooves with aligned duckbilled arches at the bottom. Though these complex structures help Nepenthes peristome mimetic surface exhibit the strongest 2D directional liquid transporting performance, its fabrication methods, such as 3D printing and semiconductor manufacturing process, is costly and inappropriate for large-area application. Therefore, the fabricating methods need further development, and nanoimprint lithography with advantages of high precision and low cost could be an effective solution.

\section{Application of bioinspired 1D and 2D un- idirectional liquid transport}

With these creative fabrication methods for bioinspired 1D directional liquid transport structures, their applications have been explored in various fields, such as fog harvesting and oil/water emulsion separation. Utilizing different responsive smart materials, the regulation of $1 \mathrm{D}$ directional liquid transport performances can be realized.

\subsection{Fog harvesting and oil/water separation}

Harvesting water directly from fog has long been considered as a potential water source for dry areas. Previous studies reveal that desert beetle's back can harvest fog with its alternating hydrophilic and hydrophobic regions, where liquid droplets in fog contact and aggregate at its hydrophilic area, and the droplets that contact the hydrophobic area would slip and bounce to the hydrophilic area ${ }^{[15,45]}$. However, the slow transporting speed and unseparated droplet covering the surface will restrict further fog deposition and lead to the de- creasing of fog harvesting rate. Therefore, quick transporting and collecting liquid droplets is critical for efficient fog harvesting. With the spider silks inspired spindle knots wire, deposited liquid droplets can be continuously and directionally transported from joints to spindle knots and spontaneously fall off the wire under gravity for further fog deposition (Fig. 12a ${ }^{[52,54]}$. It shows that larger knots size has stronger unidirectional liquid transporting ability by generating higher unbalanced Laplace pressure, thus fog harvesting rate can be increased $\sim 7$ times with spindle knots volume increasing $\sim 4$ times. Compared to the spindle knots wire, the long cone structure inspired from the cactus spine can be easily constructed into arrays on a surface to increase the fog-cone surface contacting rate ${ }^{[85]}$. The fog harvesting rate on the cone array surface can be $\sim 6$ times higher than on a smooth surface. The layout of cones also strongly affects the harvesting rate, where cones arranged in a hexagonal pattern can coagulate fog $\sim 2$ times faster than tetragonal pattern by maximizing their contact with fog flow (Fig. 12b) ${ }^{[85]}$. To further increases the surface contacting rate with fog flow, the cactus spine with smaller barbs on inspired hierarchical cone structure is an effective solution. By integrating different sized wedges and cones, a hierarchical wedged pattern and hierarchical micro-nano cone can form more trivial contact with fog flow to create higher water collecting rate (Figs. 12c and 12d). The water collection rate of hierarchical micro-nano cone can be $\sim 5$ times higher than single cactus spine ${ }^{[58,60]}$. On these hierarchical pattern and cones, liquid droplets will gradually aggregate from all these small branches to the main stem and the collecting of water will be much easier.

Similar to the air/liquid interaction in fog harvesting, 1D directional transport can also appear on air bubbles in a liquid environment. Structures including wedged patterns or sharp cones are designed, and the surface is modified into hydrophobic to form stronger bubble adhesion ${ }^{[86-88]}$. Under a similar mechanism of 1D directional liquid transport, the bubbles can be directional transport on wedged patterns or cones for air gathering or direction guiding in a liquid environment. Such manipulation of bubbles can be applied in heat transfer, renewable energy, and wastewater treatment.

Oil/water separation is an important topic since it is 


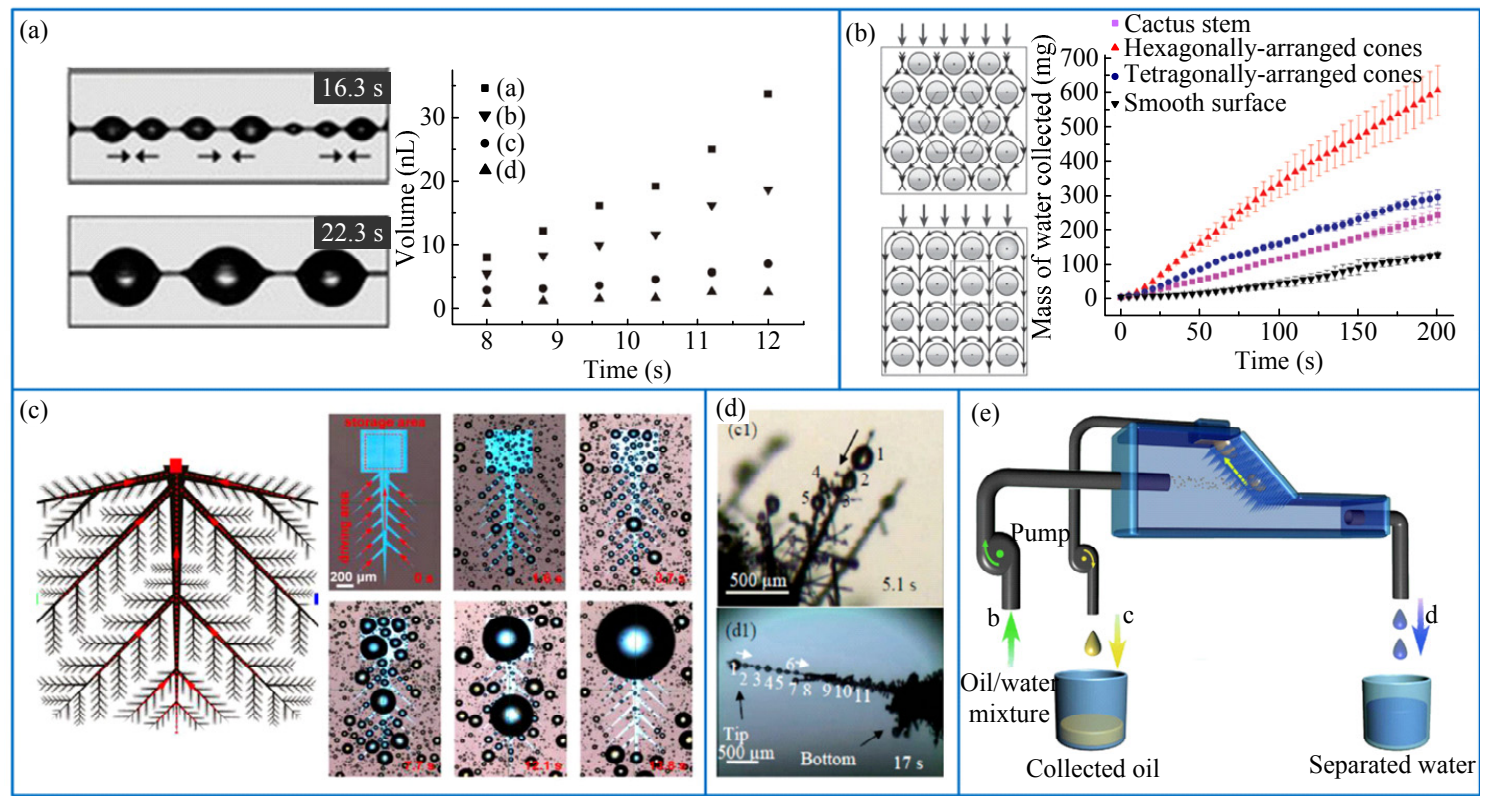

Fig. 12 (a) Fog collection on spindle knots wire. The collecting water volume increases with the knot size (reproduced from Refs. [52, 54] with permission). (b) Different layouts of cone array affect the flow of fog air and lead to different fog-cone contacting rate. The Hexagonally-arranged cone array shows the highest fog collecting speed (reproduced from Ref. [85] with permission). (c, d) Fog harvesting on hierarchical structures, including hierarchical wedged pattern and hierarchical cones (reproduced from Refs. [58,60] with permission). (e) The separation of oil/water emulsion by cone array surface. Scattered oil microdroplets deposit on cones and directionally transport to the substrate, then flows upward by floatage and aggregates into the oil layer at the top of the fluid (reproduced from Ref. [32] with permission).

critical for the oil industry and environmental protection. Normal oil/water separation is based on the phase separation strategy, that oil/water mixture will automatically separate to the upper oil layer and lower water layer by gravity in resting, and then oil/water layers can be separated by flowing the mixture through hydrophobic or oleophobic porous membranes ${ }^{[89-91]}$. However, in most cases, the mixed oil/water is in an emulsion state with micro/nano-sized droplets, which is unable to be separated by phase separation strategy. Considering the oil/water phase is similar to the water/air phase, a separation strategy for oil/water emulsion is developed inspired by the fog harvesting ability from cactus spines ${ }^{[32]}$. As shown in Fig. 12e, a cone array surface is fixed at an inclined angle. With the continuous spray of oil/water mixtures onto the cone array surface, micro-sized oil droplets are deposited on cones and directionally transported to roots by unbalanced Laplace capillary force. The oil droplets at the roots gather into larger droplets and float up to the upper layer, then, the aggregated upper layer oil is pumped out to utterly separate from water. Such cactus inspired cone array surface provides a continuous and highly efficient micro-sized oil/water separating strategy, which can be used for two incompatible liquids separation in the chemical industry or even for physiological fluids component separation in the medical field.

\subsection{Microfluidics devices}

Microfluidics has attracted worldwide attention for decades owing to its potential application in biotechnology and precision medicines. To minimize the size of the device and lower its cost, integrated and easy fabricated microfluidics are always necessary. Normally, the driving force of microfluidics is provided by off-chip modules, such as mechanical pumps, which have disadvantages like large size, pulsed flow, and high power needed. Inspired by the Nepenthes peristome, a self-pumped liquid driving system is developed with a tube that its inner surface is covered by peristome mimetic structures. When liquid fills the tube, a thin layer of liquid precursor firstly climbs up along the tube's wall, then bulk liquid gradually elevates and fills the tube. It shows the strongest liquid transporting ability that the 
height of its precursor and bulk liquid are both much higher than in microgrooves tube or smooth tube ${ }^{[92]}$. When the peristome mimetic tube is inversely immersed into a liquid bath, the structures in the tube provide a strong liquid pinning ability, so that liquid can be utterly blocked at the entrance of the tube. By bending this tube and attaching it to a tank bottom, a microsyringe with a self-pump siphon is fabricated, which can transfer a small volume of liquid under its strong unidirectional liquid transporting ability (Fig. 13a).

As one major work of microfluidics study, micro synthetic reactors can mix a small number of different solutions to produce a chemical reaction, which provides advantages including high energy efficiency, easy control of reaction speed and degree, and easily reproducible unit reactors for mass production. One key issue for designing of microreactor is to make the synthetic reaction controllable, especially the solutions' flowing speed and distribution that need to be regulated. Luckily, the 2D directional liquid transport surface offers a low cost and easy fabricated microreactor developing method. A proof-of-concept $Y$-shaped microreactor is designed by merging two-arm channels into one main channel covered by Nepenthes peristome mimetic surface ${ }^{[33]}$. When two solutions are separately dripping into each arm channels, the solutions spontaneously self-pump along the arm channels, meet at the cross-section, and continuously flow forward in the main channel along sidewalls (Fig. 13b). At the interfaces between two solutions, a chemical reaction occurs accompanied by the color of solutions gradually disappeared. The flow of solutions on the surface can be precisely adjusted by controlling the structural features of the peristome mimetic surface. This simple microreactor with of peristome mimetic surface has integrated various functions including self-pump, mixer and reactor, which provides more exquisite control of synthetic micro-reactor and offers inspiration for lap-on-chip design and fabrication.

\subsection{Self-lubricated medical devices}

With the growing demand for precision medicine, Minimally Invasive Surgery (MIS) has been highly developed owing to its advantages of small trauma, accurate operation, and quick recovery ${ }^{[93]}$. As typical surgical operations in MIS, tissue cutting and blood coagulation are usually performed with electrosurgical scalpels by electrical heating. This could easily lead to conglutination or tissue debris adhesion on the scalpel surface ${ }^{[94,95]}$, which both impede the operation of surgery and lead to potential dangers to patients. Lubricants such as silicon oil are usually covered onto the scalpels to prevent the tissue/scalpel adhesion; however, the surface could still dry out after several times of cutting due to the lack of lubricant transporting ability. Inspired from the long-term ultra-slippery liquid film on Nepenthes peristome formed by the 2D directional liquid transport, an electrosurgical scalpel has been designed and fabricated with the surface covered by gradient density micropillars along the center to edge direction ${ }^{[96]}$, which creates a gradient wettability that could continuously spread lubricant all over the scalpel surface (Fig. 14a). Through living tissue cutting tests, the bioinspired electrosurgical scalpel demonstrates superior anti-adhesion ability and better durability than traditional smooth scalpel (Fig. 14b), where the tissue adhesion force can be reduced $\sim 80 \%$ and the amount of adhered tissue debris can be decreased $\sim 88 \%$. Such self-lubricated superslippery surface could also be applied to the shell surface of endoscopy to relieve the friction against tissues.

\subsection{Self-lubricated anti-icing surfaces}

Ice accretion on engineering surfaces has been a thorny problem for many fields, such as aircraft, wind turbines, and powerlines. Lowering the ice adhesion strength on surfaces can be a reliable way to effectively decrease energy consumption for dynamic anti-icing/de-icing. Inspired by the wet peristome of $\mathrm{Ne}$ penthes, Slippery Liquid-Infused Porous Surface (SLIPS) was proposed, and lubricant film spreading in micro-structures endowed the surface with ultra-low adhesion ${ }^{[6]}$. As shown in fluorescence images, a thin water film melted at the ice-coating interface works as a lubricant to lower the ice adhesion, leading to the ice block blowing away with the airflow (Fig. 15a) ${ }^{[97]}$. Furthermore, by combining slippery lubricant film with electric heating coating, the ice adhesion strength was tremendously reduced to $\sim 3 \%$ of flat electric heating coating and $\sim 11 \%$ of superhydrophobic electric heating coating (Fig. 15b); The melted water/oil composite lubricant film also effectively reduced energy 
(a)

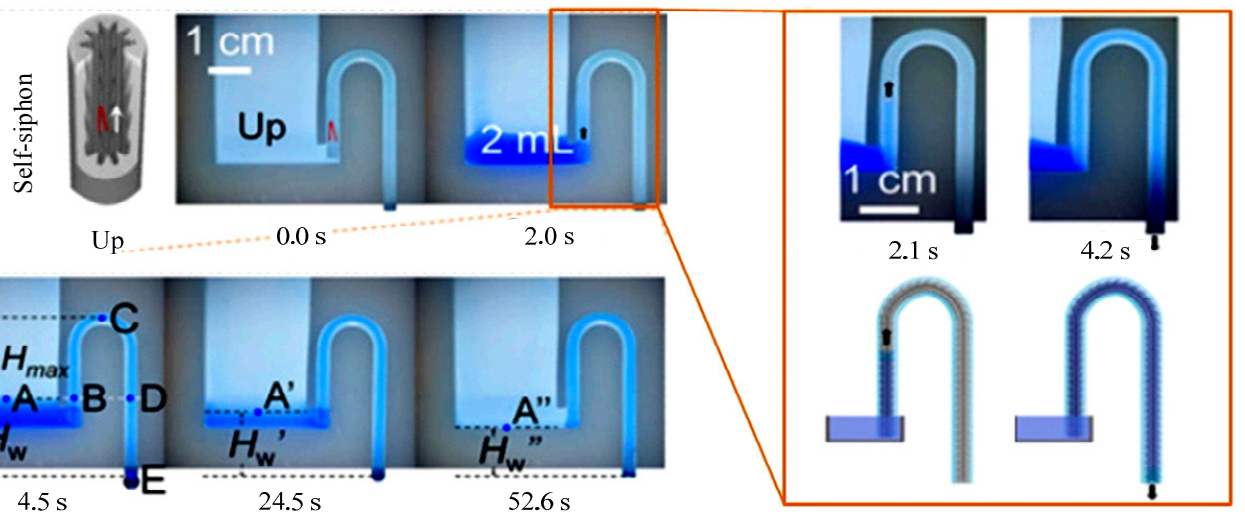

(b)

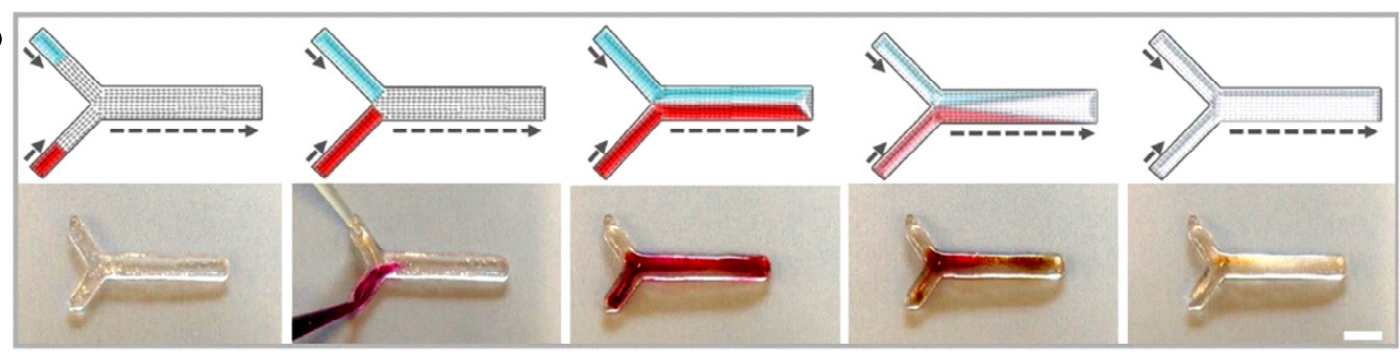

Fig. 13 (a) A self-pumped siphon inspired from Nepenthes peristome surface with the liquid directional transporting ability (reproduced from Ref. [92] with permission). (b) A self-pumped microreactor with the Nepenthes peristome mimetic surface, which can merge two solvents into a chemical reaction (reproduced from Ref. [33] with permission).

(a)

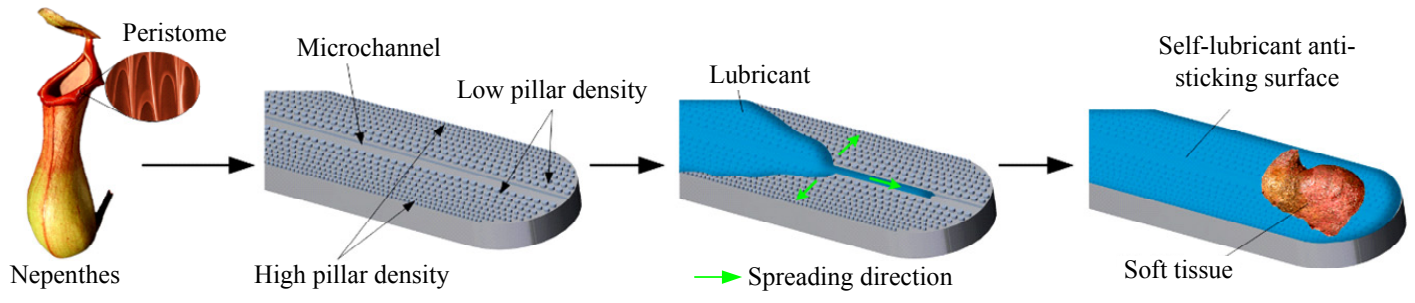

(b)

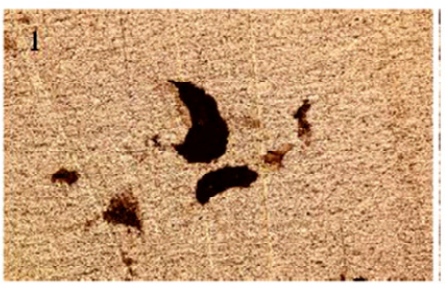

Dry smooth surface

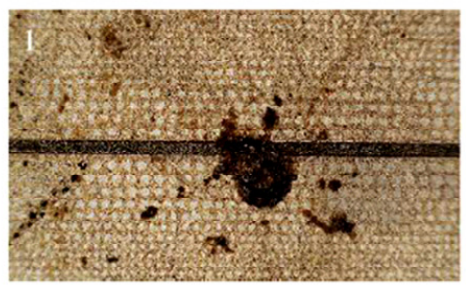

Dry gradient pillar surface

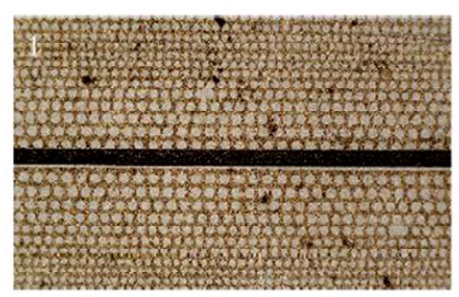

Lubricated gradient pillar surface

Fig. 14 (a) Electrosurgical scalpel surfaces with gradient density micropillars can continuously transport lubricants to tissue cutting interfaces. (b) Compared to smooth electrosurgical scalpels, the tissue stickiness can be greatly decreased on self-lubricated electrosurgical scalpels (reproduced from Ref. [96] with permission).

consumption for complete anti-icing: with the same heating power density $\left(0.58 \mathrm{~W} \cdot \mathrm{cm}^{-2}\right)$, slippery electric heating coating SEHC realized complete anti-icing while $\sim 40 \%$ of porous EHC surface was still covered by mixed ice (Fig. 15c) ${ }^{[98]}$. Generally, lubricant in SLIPS is nonrenewable and will be drained gradually, making
SLIPS incompetent to withstand dynamic icing conditions. To enhance the durability of the slippery surface against dynamic icing conditions, a lubricant supplying method is imperative. Combining Joule heat-induced water film generation and unidirectional water film transportation using Nepenthes peristome inspired 


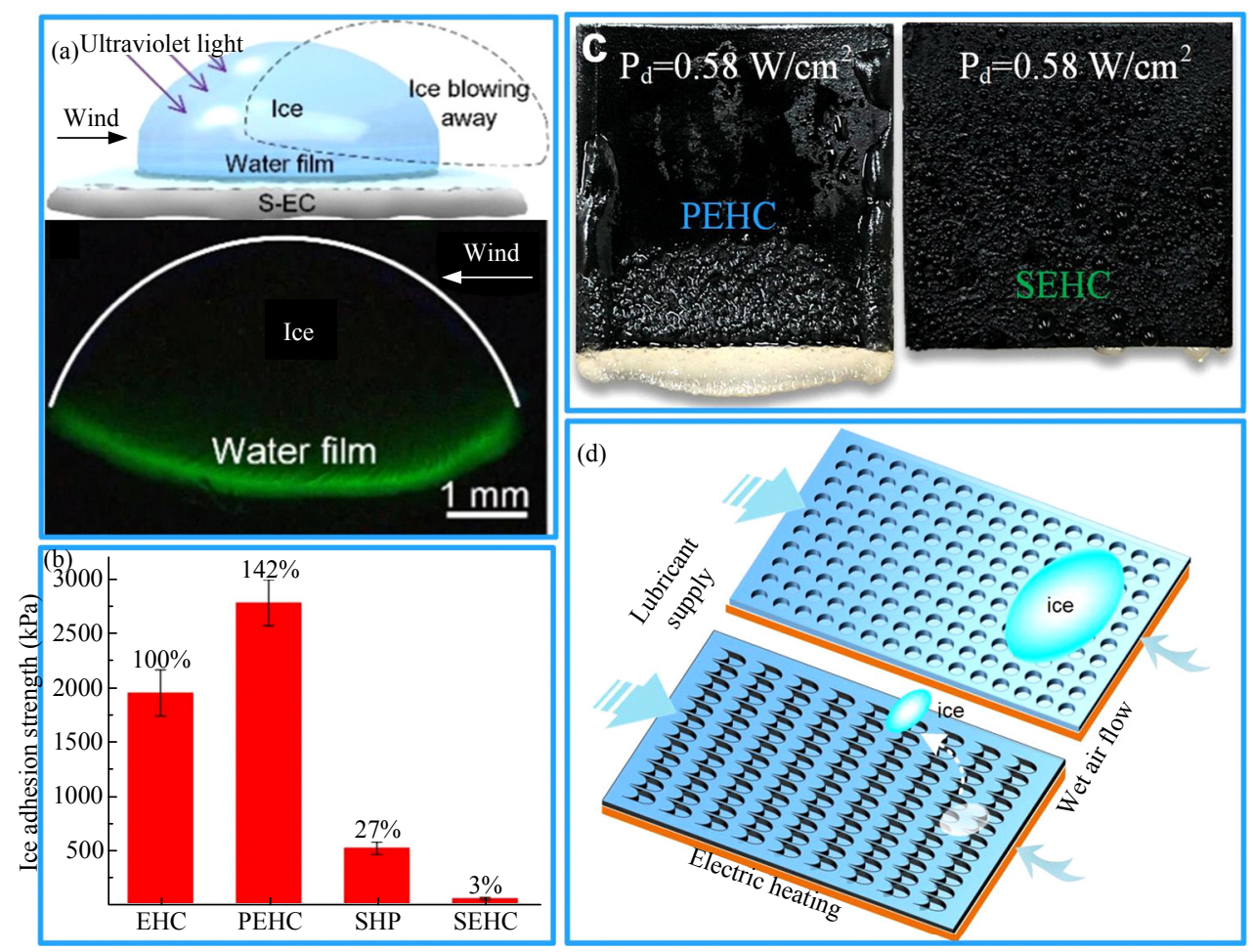

Fig. 15 Directional liquid transport for low-adhesion anti-icing applications. (a) Water film is generated by a novel electric heating coating and induces low-adhesion de-icing; as shown in the fluorescence image, a thin water film melted at the ice-coating interface works as a lubricant to lower the ice adhesion. (reproduced from Ref. [97] with permission). (b) ultra-low ice adhesion measured on Slippery oil-infused Electric Heating Coating (SEHC), only 3\% of flat Electric Heating Coating (EHC), and $11 \%$ of superhydrophobic electric heating coating (SHP). (c) the melted water/oil composite lubricant film effectively reduced energy consumption for complete anti-icing: with the same heating power density $\left(0.58 \mathrm{~W} \cdot \mathrm{cm}^{-2}\right)$, SEHC realized complete anti-icing while $\sim 40 \%$ of porous EHC surface was still covered by mixed-ice (reproduced from Ref. [98] with permission). (d) illustration of lubricant unidirectional replenishment using $\mathrm{Ne}$ penthes peristome inspired structure for durability enhancement of slippery anti-icing surfaces: by combining Joule heat-induced water film generation and unidirectional water film transportation, the liquid film can be continuously transported to the ice-surface interface to reduce energy consumption for anti-icing.

structure can be a feasible technique, by which liquid film can be continuously transported to the ice-coating interface to reduce energy consumption for anti-icing (Fig. 15d). Further investigation may focus on largescale fabrication of directional water transporting surface and its combination with Joule heat deicing materials.

\subsection{Smart materials in unidirectional liquid trans- portation}

\subsubsection{D unidirectional liquid transportation}

To make the liquid transporting performance regulatable is critical for the application of 1D unidirectional liquid transporting, such as the regulation of transporting direction or speed. Based on the revealed mechanisms in section 3.1, it is possible to adjust direc- tional liquid transport by tuning the surface wettability or changing the shape of the 1D structure. Accompanied by the significant development of smart materials, the regulation of $1 \mathrm{D}$ directional liquid transport can be realized with various trigging methods, such as thermal response, light response, or magnetic response.

As a thermal responsive material, $N$-isopropyl acrylamide (NIPAAm) polymer, its contact angles can be changed from hydrophilic $51.64^{\circ}$ to hydrophobic $107.09^{\circ}$ with temperature increasing from $20{ }^{\circ} \mathrm{C}$ to $45^{\circ} \mathrm{C}^{[99]}$. By dip-coating carbon fiber in NIPPAAm, a bioinspired spindle-knots wire is achieved with thermal controlling ability. At a temperature of $25{ }^{\circ} \mathrm{C}$, the hydrophilic wire's spindle knots show stronger Laplace pressure, which directionally drives liquid from joints to spindle knots (Fig. 16a). With the temperature rising 


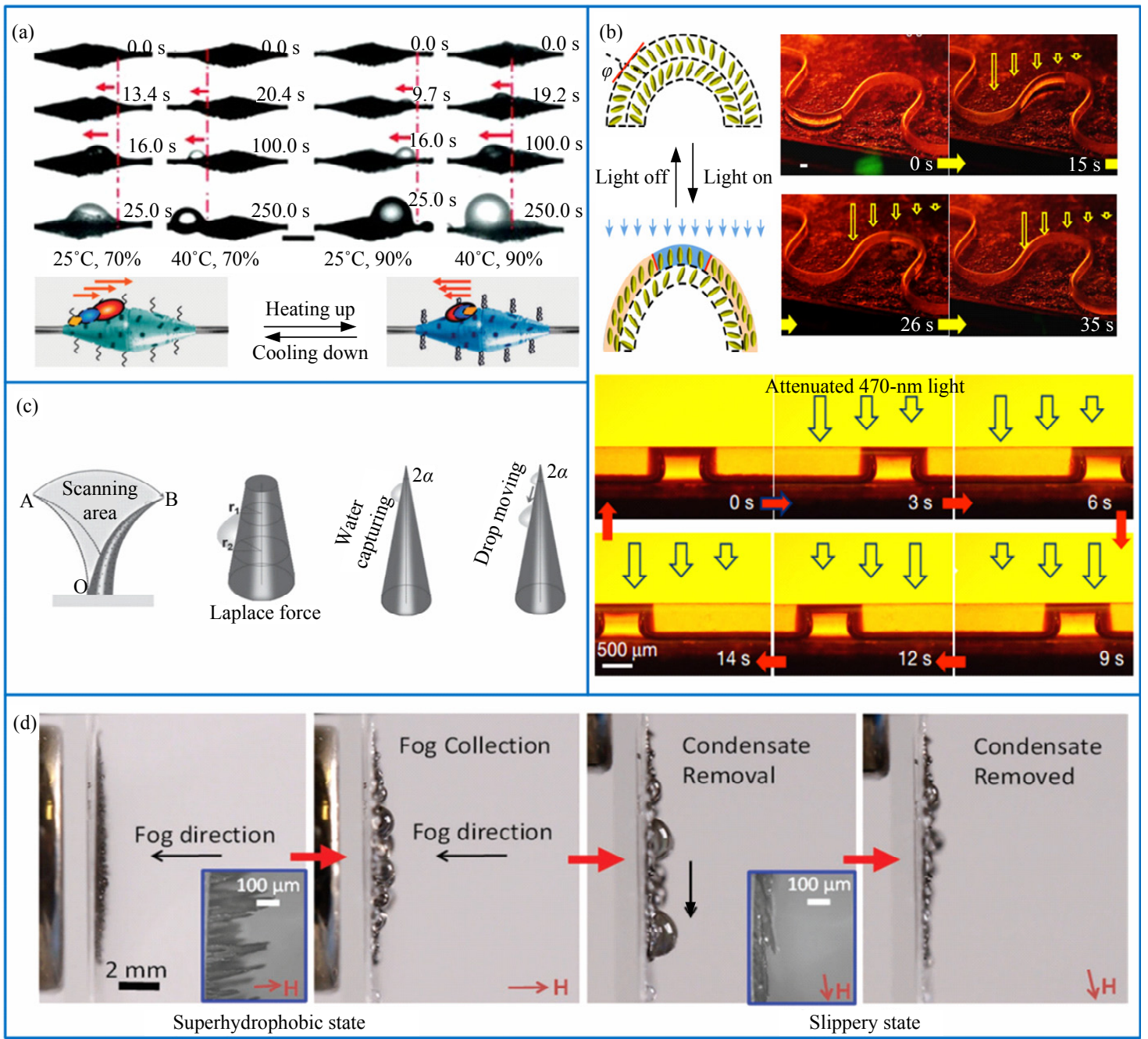

Fig. 16 (a) With thermal responsive coating, the liquid transport direction on spindle knots wire can be controlled by adjusting the environment temperature (reproduced from Ref. [99] with permission). (b) A capillary tube fabricated by photodeformable material can be shaped into an asymmetric cone tube under gradient light intensity, which leads to liquid directional transport in the tube. The liquid flow direction can be regulated by changing the gradient of light intensity (reproduced from Ref. [34] with permission). (c) Cones embedded with ferromagnetic particles exhibit a swing motion under a shifting magnetic field, which could enhance the fog-cone surface contact rate to achieve a higher water collecting ratio even in static air (reproduced from Ref. [27] with permission). (d) Fabricated with magnetic response material, the cone array can be bent for about $90^{\circ}$ to clear away the deposited water droplets for the next round of water collecting (reproduced from Ref. [31] with permission).

to $40{ }^{\circ} \mathrm{C}$, the spindle knots wire changes into hydrophobic and the direction of Laplace pressure shifts to the joint side, thus, the liquid transport direction is inverted.

The liquid directional transport direction can also be controlled by adjusting the asymmetry of 1D structures. As shown in Fig. 16b, an isometric capillary tube is fabricated with a light-deformable material, i.e. linear liquid crystal polymer, which can be polarized by light to generate internal stress to create deformation ${ }^{[34]}$. Through light irradiation with gradient intensity, the isometric tube changes into an asymmetric cone tube, and liquid directional flows the cone tip side. By changing the strength of light intensity or switching the direction of the light gradient, the asymmetry of the cone tube can be precisely regulated, and the liquid flow speed and direction can be controlled. Such a photodeformable tube shows perfect durability that can function even after over 100 cycles of light-induced deformation. With this easy adjustable light-controlling method, the 1D directional liquid transport tube can realize various functions, such as lifting liquid upward against gravity, 
guiding liquid in $\mathrm{S}$ shaped tubes, or mixing different liquids in Y shaped tubes.

As described in section 3.3.1, fog harvesting is an important application for 1D unidirectional liquid transport, and various spindle knot wires or cones array surfaces are fabricated and validated for fog harvesting application. However, these researches are performed under flowing fog conditions with microdroplets in the air continuously collide onto the cone surface. Since natural fog mainly exits in the static air, the contact between fog air and cones will be greatly declined, which results in an extremely low fog harvesting rate in nature. To solve this problem, Peng et al. have introduced a smart cone array surface fabricated by a magnetic response material, where the cones can bend under different magnetic field ${ }^{[27]}$. This material is a composition of polymers and micro/nano ferromagnetic particles. Under a shifting magnetic field, the cones can be driven repeatedly swinging from side to side to maximize their contact with fog (Fig. 16c). The water harvesting rate is greatly enhanced even in a static fog environment, which can be $\sim 50$ times higher than on static cones. Another challenge for fog harvesting is to quickly separate the collected water to clear space for further fog deposition. Huang et al. have fabricated a magnetic response cone array surface, where the cones can bend to $\sim 90^{\circ}$ under a controlled magnetic field to change the surface into the liquid slippery state ${ }^{[31]}$. With this specialty, the fog harvesting is composed of two processes, i.e. cones straighten up-right to quickly collect fog, and cones bent $\sim 90^{\circ}$ to easily separate collected water from the surface by gravity (Fig. 16d). With this process continuing, fog can be successively turned into water and efficiently collected by a container with little energy input. The integrations of smart materials into 1D directional liquid transport open a new way for liquid regulation in medical and sensor fields.

\subsubsection{D unidirectional liquid transportation}

To further expand the application of bioinspired 2D unidirectional liquid transport surfaces, its liquid transporting ability needs to be adjustable, such as the anisotropy of liquid transporting, transporting distance, and transporting speed. Based on the 2D liquid directional transport mechanisms, the adjustment of liquid spread- ing properties can be realized by regulating surface wettability or feature structures. With the emerging of various types of smart materials, controlling strategies including magnetism, heat, or light are introduced to regulate the $2 \mathrm{D}$ directional liquid transport on both inclined pillar arrays surfaces and Nepenthes peristome mimetic surfaces.

For a 2D directional liquid transport surface constructed with inclined pillar arrays, the liquid is dragged forward to pillar inclined direction by capillary attraction and pinned in the opposite direction. A larger pillar inclined angle can lead to a stronger forward dragging force and higher backward pinning effect, which leads to stronger anisotropic liquid transporting performance. A 2D controllable directional liquid transport surface can be achieved by fabricating the pillars with ferromagnetic materials, such as electroplating ferromagnetic nick$\mathrm{el}^{[100]}$, or mixing prepolymer with magnetic particles ${ }^{[29]}$. Under an inclined magnetic field, the pillars demonstrate uniform inclination, and the inclined angle can be adjusted from $-90^{\circ}$ to $+90^{\circ}$ by manipulating the direction of the magnetic field. On such surface, liquid transport can be real-time controlled among the states of spreading, pausing, or direction shifting, as shown in Fig. 17a. Since the direction of the magnetic field can be rotated $360^{\circ}$, a liquid distributor is constructed by radially distributing channels around the magnetic responsive pillar arrays surface. By controlling the direction of the magnetic field, different liquids can be efficiently separated into chosen channels (Fig. 17b).

Another 2D controllable unidirectional liquid transport is based on the Nepenthes peristome surface, which has been broadly studied because of its strong directional transporting ability with long-distance and high speed. Since the directional liquid transport is formed by superhydrophilic property and duckbilled structure, controlling strategies mainly focus on regulating the surface's wettability or micro-structures. By grafting the thermal-responsive PNIPAM onto the artificial peristome mimetic surface, it shows 2D temperature-controlled directional water transporting, that water directionally transports at a temperature of $\sim 20^{\circ} \mathrm{C}$, while pinned in stop transporting at a temperature of $\sim 40{ }^{\circ} \mathrm{C}$ (Fig. 18a) ${ }^{[77]}$.

Since common hydrophilic materials are 

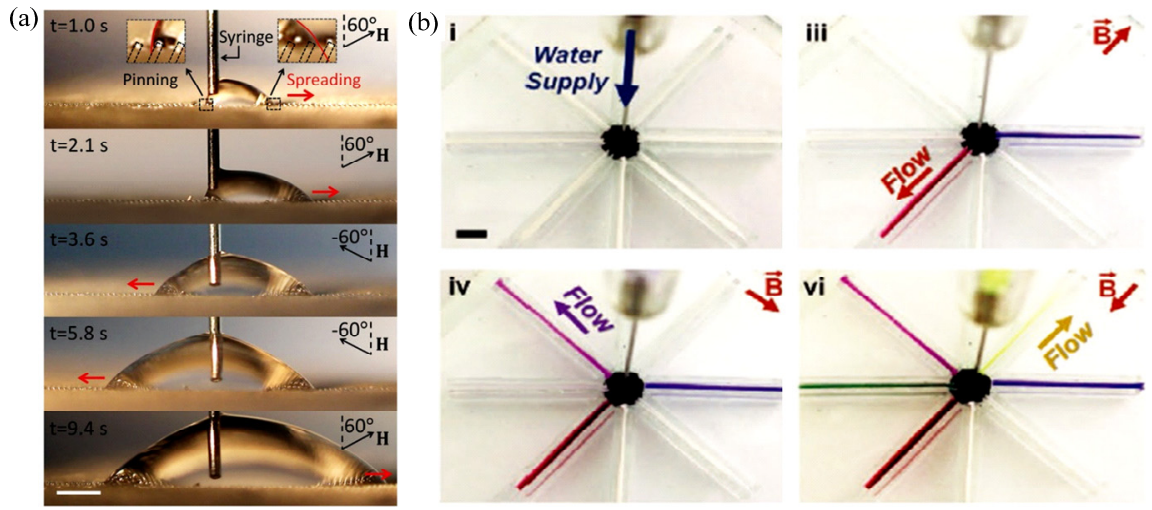

Fig. 17 (a, b) By fabricating the pillar with magnetic response material, its inclination can be controlled by the magnetic field with different directions, then, the liquid's flow can be adjusted by changing the pillar's inclined direction or inclined angle (reproduced from Ref. $[29,100]$ with permission).

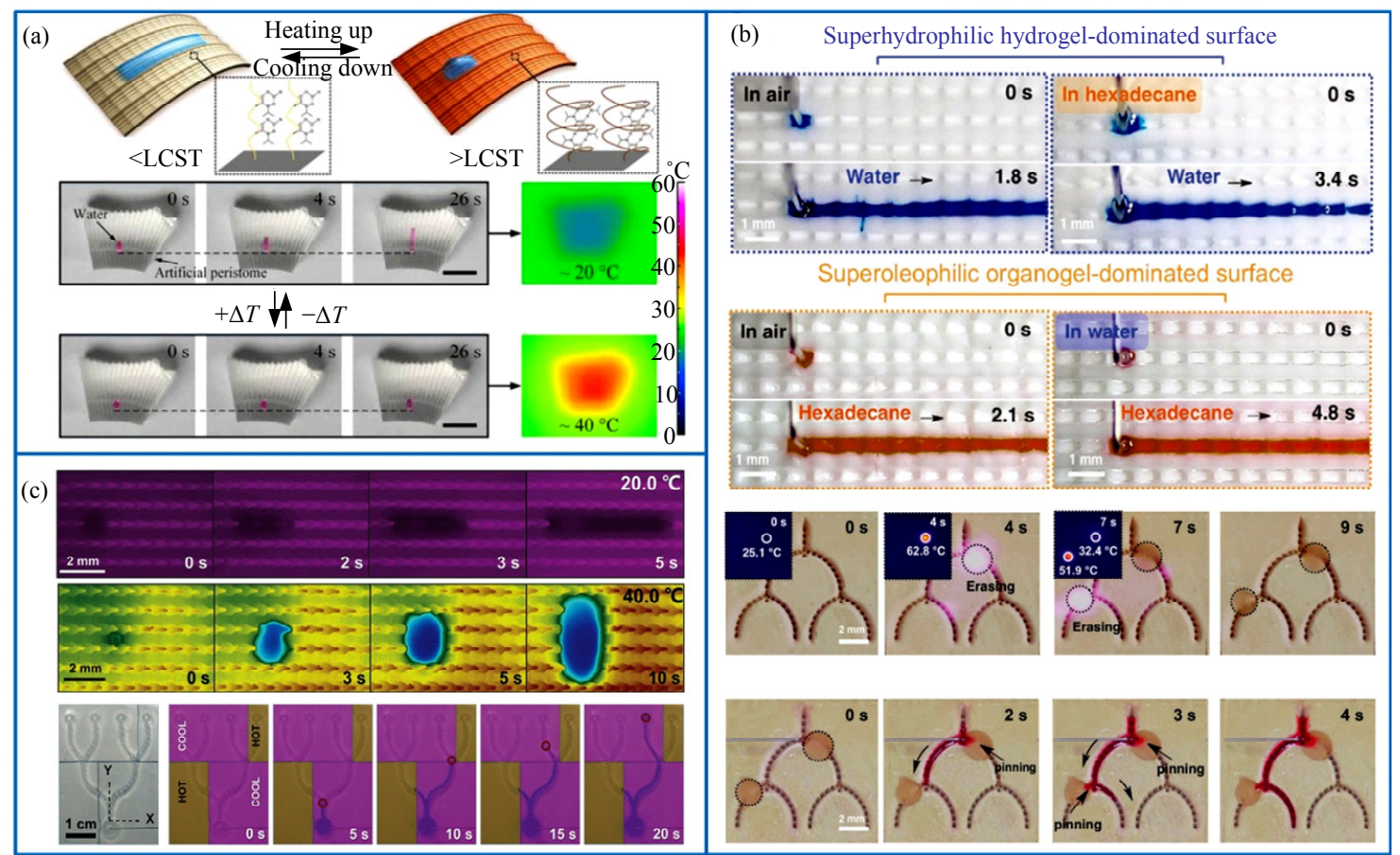

Fig. 18 (a) With a temperature responsive coating, the directional liquid transporting ability on Nepenthes peristome mimetic surface can be switched on/off by changing the temperature to adjust the surface wettability (reproduced from Ref. [77] with permission). (b) Super-amphiphilic material with switchable hydrophilic and oleophilic ability can directionally transport water or organic liquid after switching its surface wettability. The material is also thermal-deformable so that the shape of channels can be regulated by infrared LED irradiation to switch on/off of liquid transporting (reproduced from Ref. [14] with permission). (c) A Nepenthes peristome mimetic surface with duckbilled micro-cavities fabricated by a thermal-deformable material. Liquid transport can be changed from unidirectional to bidirectional (reproduced from Ref. [35]with permission).

oleophobic to organic liquid, a Nepenthes peristome mimetic is unable to directional transport water and organic at the same time. By introducing heteronetwork organohydrogels with rapid super-amphiphilicity altering ability, the peristome mimetic surface is fabricated with both water and organic liquid directional transporting ability (Fig. 18b, upper) ${ }^{[14]}$. This material also possesses a reconfigurable surface topography in response to temperature. By mixing the material with photothermal responsive $\mathrm{Fe}_{3} \mathrm{O}_{4}$ nanoparticles, the mi- 
crogroove structure of the bioinspired surface can be reshaped by infrared LED light, and the flow of liquid in channels can be controlled (Fig. 18b, lower). With thermal shrink gel to fabricate the duckbilled cavity on the peristome surface, the liquid directional transport can be switched on/off by opening or closing the duckbilled cavity with the control of surface temperature (Fig. 18c $)^{[35]}$. After placing this surface on a substrate with arrayed cool and hot patterns, the liquid flow on the surface can be adjusted to selected channels, and a programable liquid transport surface is developed. Based on a 2D unidirectional liquid transport surface, the integration with smart materials provides diversified liquid regulation methods and widens its potential application in various fields such as microfluidics and biomedical devices.

Currently, various in situ trigging methods have been introduced, including temperature, light, magnetic field, and electric field. Combing these trigging methods could realize more complex liquid adjustments and create multifunction. Besides, accurate controlling methods are important for liquid adjustment. The magnetic or electric fields with directionality and precise adjustability could be ideal precise controlling methods. For example, with magnetic response material fabricated inclined pillar array surface, the direction of pillar inclining can be adjusted $360^{\circ}$ and the inclined angle can be tuned precisely, thus liquid on such surface can be accurately directed to any direction with controlled speed, which provides potential applications for liquid delivering or splitting. Applying smart materials into 1D and 2D directional liquid transport could greatly expand their application in cell screening, liquid chromatography, and fuel cells.

\section{Conclusions and perspectives}

As summarized above, significant achievements have been made over the past decades in exploring nature samples with 1D and 2D unidirectional liquid transport. Their underlying mechanisms have been revealed under the cooperative effects of $1 \mathrm{D} / 2 \mathrm{D}$ featured micro-nano hierarchical structures and tuned material wettability. Based on these mechanisms, bioinspired 1D and 2D directional liquid transport cones and surfaces have been constructed by numerous novel fabrication methods and surface modification strategies. Such bioinspired 1D and 2D materials demonstrate similar or even better directional liquid transporting ability than nature samples, which provides potential applications in renewable energy and biomedical engineering fields, such as fog collection, oil/water separation, microreactors, microfluidics, and biomedical devices. Additionally, integrating with responsive materials, 1D and 2D smart directional transport cones or surfaces have been created with tunable featured structures or material wettability that more complex and programable regulations are established in liquid transport.

Despite these significant achievements in bioinspired directional liquid transport, there are still many challenges that need to be resolved. The liquid directional transport on nature samples needs to be continuously explored from the sky to the water, and more advanced characterization methods should be built for observing natural samples with faster speed, longer distance, and larger liquid transport volume rate. The fabrication of bioinspired directional liquid transport materials needs to be further improved and developed with a performance at low cost, large-area, and high durability. Their applications could be expanded to many other fields, such as cell screening in biomedicine, body fluid self-pumping in wearable sensors, and power generation for micro-energy. Moreover, 1D and 2D liquid transport exist not only in macro/micro scales as described in the above sections but also in nano or even quantum scales, such as liquid flowing in carbon nanotubes ${ }^{[101-104]}$ or ions penetrating biological membranes. Exploration of liquid transport at these scales is of importance for both revealing its mechanisms and applications in biological systems. With the increasing discovery of nature and advancing of fabrication, it is believed that the development of bioinspired unidirectional liquid transport could meet a new outbreak in the near future.

\section{Acknowledgment}

We thank the National Key R\&D Program of China (No. 2019YFB1309702), and the National Natural Science Foundation of China (Nos. 51935001, 51725501 and 51905022). We also thank M. Li, G. Wang and Y. Lai from the National Natural Science Founda- 
tion of China for their support and helpful discussions.

Open Access This article is licensed under a Creative Commons Attribution 4.0 International License, which permits use, sharing, adaptation, distribution and reproduction in any medium or format, as long as you give appropriate credit to the original author(s) and the source, provide a link to the Creative Commons licence, and indicate if changes were made.

The images or other third party material in this article are included in the article's Creative Commons licence, unless indicated otherwise in a credit line to the material. If material is not included in the article's Creative Commons licence and your intended use is not permitted by statutory regulation or exceeds the permitted use, you will need to obtain permission directly from the copyright holder.

To view a copy of this licence, visit http://creativecommons.org/licenses/by/4.0/.

\section{References}

[1] $\mathrm{Xu} \mathrm{T}$, Lin Y C, Zhang M X, Shi W W, Zheng Y M. High-efficiency fog collector: Water unidirectional transport on heterogeneous rough conical wires. ACS Nano, 2016, 10, 10681-10688.

[2] Wang T Q, Chen H X, Liu K, Li Y, Xue P H, Yu Y, Wang S L, Zhang J H, Kumacheva E, Yang B. Anisotropic Janus Si nanopillar arrays as a microfluidic one-way valve for gas-liquid separation. Nanoscale, 2014, 6, 3846-3853.

[3] Shang L R, Cheng Y, Zhao Y J. Emerging droplet microfluidics. Chemical Reviews, 2017, 117, 7964-8040.

[4] Ware C S, Smith-Palmer T, Peppou-Chapman S, Scarratt L R J, Humphries E M, Balzer D, Neto C. Marine antifouling behavior of lubricant-infused nanowrinkled polymeric surfaces. ACS Applied Materials \& Interfaces, 2018, 10, 4173-4182.

[5] Jokinen V, Kankuri E, Hoshian S, Franssila S, Ras R H A. Superhydrophobic blood - Repellent surfaces. Advanced Materials, 2018, 30, 1705104.

[6] Wong T S, Kang S H, Tang S K Y, Smythe E J, Hatton B D, Grinthal A, Aizenberg J. Bioinspired self-repairing slippery surfaces with pressure-stable omniphobicity. Nature, 2011, 477, 443-447.

[7] Zhang P F, Chen H W, Zhang L W, Ran T, Zhang D Y. Transparent self-cleaning lubricant-infused surfaces made with large-area breath figure patterns. Applied Surface Science, 2015, 355, 1083-1090.
[8] Epstein A K, Wong T S, Belisle R A, Boggs E M, Aizenberg J. Liquid-infused structured surfaces with exceptional anti-biofouling performance. Proceedings of the National Academy of Sciences of the United States of America, 2012, 109, 13182-13187.

[9] He M, Zhang Q L, Zeng X P, Cui D P, Chen J, Li H L, Wang J J, Song Y L. Hierarchical porous surface for efficiently controlling microdroplets' self-removal. Advanced Materials, 2013, 25, 2291-2295.

[10] Liu K S, Jiang L. Bio-inspired self-cleaning surfaces. Annual Review of Materials Research, 2012, 42, 231-263.

[11] Nishimoto S, Bhushan B. Bioinspired self-cleaning surfaces with superhydrophobicity, superoleophobicity, and superhydrophilicity. RSC Advances, 2013, 3, 671-690.

[12] Li D K, Wang Z T, Wu D H, Han G C, Guo Z G. A hybrid bioinspired fiber trichome with special wettability for water collection, friction reduction and self-cleaning. Nanoscale, 2019, 11, 11774-11781.

[13] Yu C L, Li C X, Gao C, Dong Z C, Wu L, Jiang L. Time-dependent liquid transport on a biomimetic topological surface. ACS Nano, 2018, 12, 5149-5157.

[14] Zhao Z G, Li C X, Dong Z C, Yang Y C, Zhang L H, Zhuo S Y, Zhou X T, Xu Y C, Jiang L, Liu M J. Adaptive superamphiphilic organohydrogels with reconfigurable surface topography for programming unidirectional liquid transport. Advanced Functional Materials, 2019, 29, 1807858.

[15] Parker A R, Lawrence C R. Water capture by a desert beetle. Nature, 2001, 414, 33-34.

[16] Feng L, Li S H, Li Y S, Li H J, Zhang L J, Zhai J, Song Y L, Liu B Q, Jiang L, Zhu D B. Super-hydrophobic surfaces: From natural to artificial. Advanced Materials, 2002, 14, 1857-1860.

[17] Prakash M, Quere D, Bush J W M. Surface tension transport of prey by feeding shorebirds: The capillary ratchet. Science, 2008, 320, 931-934.

[18] Reis P M, Jung S H, Aristoff J M, Stocker R. How cats lap: Water uptake by felis catus. Science, 2010, 330, 1231-1234.

[19] Zheng Y M, Bai H, Huang Z B, Tian X L, Nie F Q, Zhao Y, Zhai J, Jiang L. Directional water collection on wetted spider silk. Nature, 2010, 463, 640-643.

[20] Ju J, Bai H, Zheng Y M, Zhao T Y, Fang R C, Jiang L. A multi-structural and multi-functional integrated fog collection system in cactus. Nature Communications, 2012, 3, 1247.

[21] Ishii D, Horiguchi H, Hirai Y, Yabu H, Matsuo Y, Ijiro K, Tsujii K, Shimozawa T, Hariyama T, Shimomura M. Water transport mechanism through open capillaries analyzed by 
direct surface modifications on biological surfaces. Scientific Reports, 2013, 3, 3024.

[22] Wong T S, Sun T L, Feng L, Aizenberg J. Interfacial materials with special wettability. MRS Bulletin, 2013, 38, 366-371.

[23] Liu C C, Zhu J, Zheng Y M, Jiang L. Asymmetric ratchet effect for directional transport of fog drops on static and dynamic butterfly wings. ACS Nano, 2014, 8, 1321-1329.

[24] Chen H W, Ran T, Gan Y, Zhou J J, Zhang Y, Zhang L W, Zhang D Y, Jiang L. Ultrafast water harvesting and transport in hierarchical microchannels. Nature Materials, 2018, 17, 935-942.

[25] Chen H W, Zhang P F, Zhang L W, Liu H L, Jiang Y, Zhang D Y, Han Z W, Jiang L. Continuous directional water transport on the peristome surface of Nepenthes alata. $\mathrm{Na}$ ture, 2016, 532, 85-89.

[26] Song Y Y, Liu Y, Jiang H B, Li S Y, Kaya C, Stegmaier T, Han Z W, Ren L Q. Bioinspired Fabrication of one dimensional graphene fiber with collection of droplets application. Scientific Reports, 2017, 7, 12056.

[27] Peng Y, He Y X, Yang S, Ben S, Cao M Y, Li K, Liu K S, Jiang L. Magnetically induced fog harvesting via flexible conical arrays. Advanced Functional Materials, 2015, 25, 5967-5971.

[28] Ju J, Xiao K, Yao X, Bai H, Jiang L. Bioinspired conical copper wire with gradient wettability for continuous and efficient fog collection. Advanced Materials, 2013, 25, 5937-5942.

[29] Cao M Y, Jin X, Peng Y, Yu C M, Li K, Liu K S, Jiang L. Unidirectional wetting properties on multi-bioinspired magnetocontrollable slippery microcilia. Advanced Materials, 2017, 29, 1606869.

[30] Chen H W, Zhang L W, Zhang P F, Zhang D Y, Han Z W, Jiang L. A novel bioinspired continuous unidirectional liquid spreading surface structure from the peristome surface of Nepenthes alata. Small, 2017, 13, 1601676.

[31] Huang Y, Stogin B B, Sun N, Wang J, Yang S K, Wong T S. A switchable cross-species liquid repellent surface. $A d$ vanced Materials, 2017, 29, 1604641.

[32] Li K, Ju J, Xue Z X, Ma J, Feng L, Gao S, Jiang L. Structured cone arrays for continuous and effective collection of micron-sized oil droplets from water. Nature Communications, 2013, 4, 3276.

[33] Shang L R, Yu Y R, Gao W, Wang Y T, Qu L L, Zhao Z, Chai R J, Zhao Y J. Bio-inspired anisotropic wettability surfaces from dynamic ferrofluid assembled templates. Advanced Functional Materials, 2018, 28, 1705802.
[34] Lv J A, Liu Y Y, Wei J, Chen E Q, Qin L, Yu Y L. Photocontrol of fluid slugs in liquid crystal polymer microactuators. Nature, 2016, 537, 179-184.

[35] Li C X, Yu C L, Hao D Z, Wu L, Dong Z C, Jiang L. Smart liquid transport on dual biomimetic surface via temperature fluctuation control. Advanced Functional Materials, 2018, 28, 1707490.

[36] Malvadkar N A, Hancock M J, Sekeroglu K, Dressick W J, Demirel M C. An engineered anisotropic nanofilm with unidirectional wetting properties. Nature Materials, 2010, 9, 1023-1028.

[37] Hancock M J, Sekeroglu K, Demirel M C. Bioinspired directional surfaces for adhesion, wetting and transport. Advanced Functional Materials, 2012, 22, 2223-2234.

[38] Sekeroglu K, Demirel M C. A fluidic device with polymeric textured ratchets. Polymer (Guildf), 2015, 58, 30-35.

[39] Lorenceau E, Quere D. Drops on a conical wire. Journal of Fluid Mechanics, 2004, 510, 29-45.

[40] Renvoisé P, Bush J, Prakash M, Quéré D. Drop propulsion in tapered tubes. EPL (Europhysics Letters), 2009, 86, 64003.

[41] Xu W, Lan Z, Peng B L, Wen R F, Chen Y S, Ma X H. Directional movement of droplets in grooves: Suspended or immersed? Scientific Reports, 2016, 6, 18836.

[42] Wang Q B, Su B, Liu H, Jiang L. Chinese brushes: Controllable liquid transfer in ratchet conical hairs. Advanced Materials, 2014, 26, 4889-4894.

[43] Wang Q B, Yao X, Liu H, Quere D, Jiang L. Self-removal of condensed water on the legs of water striders. PNAS, 2015, 112, 9247-9252.

[44] Chaudhury M K, Whitesides G M. How to make water run uphill. Science, 1992, 256, 1539-1541.

[45] Daniel S, Chaudhury M K, Chen J C. Fast drop movements resulting from the phase change on a gradient surface. Science, 2001, 291, 633-636.

[46] Fang G P, Li W, Wang X F, Qiao G J. Droplet motion on designed microtextured superhydrophobic surfaces with tunable wettability. Langmuir, 2008, 24, 11651-11660.

[47] Choi W, Tuteja A, Mabry J M, Cohen R E, McKinley G H. A modified Cassie-Baxter relationship to explain contact angle hysteresis and anisotropy on non-wetting textured surfaces. Journal of Colloid and Interface Science, 2009, 339, 208-216.

[48] Yang J T, Yang Z H, Chen C Y, Yao D J. Conversion of surface energy and manipulation of a single droplet across micropatterned surfaces. Langmuir, 2008, 24, 9889-9897.

[49] Wenzel R N. Resistance of solid surfaces to wetting by water. Industrial \& Engineering Chemistry, 1936, 28, 988-994. 
[50] Cassie A B D, Baxter S. Wettability of porous surfaces. Transactions of the Faraday Society, 1944, 40, 546-551.

[51] Feng S L, Hou Y P, Chen Y, Xue Y, Zheng Y M, Jiang L. Water-assisted fabrication of porous bead-on-string fibers. Journal of Materials Chemistry A, 2013, 1, 8363-8366.

[52] Bai H, Tian X L, Zheng Y M, Ju J, Zhao Y, Jiang L. Direction controlled driving of tiny water drops on bioinspired artificial spider silks. Advanced Materials, 2010, 22, $5521-5525$.

[53] Shang L R, Fu F F, Cheng Y, Yu Y R, Wang J, Gu Z Z, Zhao Y J. Bioinspired multifunctional spindle-knotted microfibers from microfluidics. Small, 2017, 13, 1600286.

[54] Bai H, Ju J, Sun R Z, Chen Y, Zheng Y M, Jiang L. Controlled fabrication and water collection ability of bioinspired artificial spider silks. Advanced Materials, 2011, 23, 3708-3711.

[55] Chen Y, Wang L, Xue Y, Jiang L, Zheng Y M. Bioinspired tilt-angle fabricated structure gradient fibers: Micro-drops fast transport in a long-distance. Scientific Reports, 2013, 3, 2927.

[56] Feng S L, Wang Q Q, Xing Y, Hou Y P, Zheng Y M. Continuous directional water transport on integrating tapered surfaces. Advanced Materials Interfaces, 2020, 7, 2000081.

[57] Bai H, Wang L, Ju J, Sun R Z, Zheng Y M, Jiang L. Efficient water collection on integrative bioinspired surfaces with star-shaped wettability patterns. Advanced Materials, 2014, 26, 5025-5030.

[58] Wang M, Liu Q, Zhang H R, Wang C, Wang L, Xiang B X, Fan Y T, Guo C F, Ruan S C. Laser direct writing of tree-shaped hierarchical cones on a superhydrophobic film for high-efficiency water collection. ACS Applied Materials \& Interfaces, 2017, 9, 29248-29254.

[59] Cao M Y, Ju J, Li K, Dou S X, Liu K S, Jiang L. Facile and large-scale fabrication of a cactus-inspired continuous fog collector. Advanced Functional Materials, 2014, 24, $3235-3240$.

[60] Heng X, Xiang M M, Lu Z H, Luo C. Branched ZnO wire structures for water collection inspired by cacti. ACS Applied Materials \& Interfaces, 2014, 6, 8032-8041.

[61] Park K C, Kim P, Grinthal A, He N, Fox D, Weaver J C, Aizenberg J. Condensation on slippery asymmetric bumps. Nature, 2016, 531, 78-82.

[62] Deng S L, Shang W F, Feng S L, Zhu S P, Xing Y, Li D, Hou Y P, Zheng Y M. Controlled droplet transport to target on a high adhesion surface with multi-gradients. Scientific Reports, 2017, 7, 45687.

[63] Chu K H, Xiao R, Wang E N. Uni-directional liquid spreading on asymmetric nanostructured surfaces. Nature Materials, 2010, 9, 413-417.

[64] Higuera F, Medina A, Linan A. Capillary rise of a liquid between two vertical plates making a small angle. Physics of Fluids, 2008, 20, 102102.

[65] Ponomarenko A, Quéré D, Clanet C. A universal law for capillary rise in corners. Journal of Fluid Mechanics, 2011, 666, 146-154.

[66] Oliver J F, Huh C, Mason S G. Resistance to spreading of liquids by sharp edges. Journal of Colloid and Interface Science, 1977, 59, 568-581.

[67] Feng S L, Wang S J, Gao L C, Li G J, Hou Y P, Zheng Y M. Controlled directional water-droplet spreading on a high-adhesion surface. Angewandte Chemie International Edition, 2014, 53, 6163-6167.

[68] Garrod R P, Harris L G, Schofield W C, McGettrick J, Ward L J, Teare D O, Badyal J P. Mimicking a Stenocara beetle's back for microcondensation using plasmachemical patterned superhydrophobic-superhydrophilic surfaces. Langmuir, 2007, 23, 689-693.

[69] Zhai L, Berg M C, Cebeci F C, Kim Y, Milwid J M, Rubner M F, Cohen R E. Patterned superhydrophobic surfaces: Toward a synthetic mimic of the namib desert beetle. Nano Letters, 2006, 6, 1213-1217.

[70] Liu C R, Sun J, Li J, Xiang C H, Che L F, Wang Z K, Zhou X F. Long-range spontaneous droplet self-propulsion on wettability gradient surfaces. Scientific Reports, 2017, 7, 7552.

[71] Li J, Qin Q H, Shah A, Ras R H, Tian X, Jokinen V. Oil droplet self-transportation on oleophobic surfaces. Science Advances, 2016, 2, e1600148.

[72] Kumar M, Bhardwaj R, Sahu K C. Wetting dynamics of a water droplet on micropillar surfaces with radially varying pitches. Langmuir, 2020, 36, 5312-5323.

[73] Geng H, Bai H Y, Fan Y Y, Wang S Y, Ba T, Yu C M, Cao M Y, Jiang L. Unidirectional water delivery on a superhydrophilic surface with two-dimensional asymmetrical wettability barriers. Materials Horizons, 2018, 5, 303-308.

[74] Muto K, Ishii D. Effects of anisotropic liquid spreading on liquid transport in arrow-like micropillar arrays. Colloids and Surfaces A: Physicochemical and Engineering Aspects, 2018, 544, 86-90.

[75] Lin Y C, Hu Z Y, Gao C L, Guo Z Y, Li C, Zheng Y M. Directional droplet spreading transport controlled on tilt Angle pillar arrays. Advanced Materials Interfaces, 2018, 5, 1800962.

[76] Wang Y, Gao Y, Wyss H M, Anderson P D, Toonder J M J D. Artificial cilia fabricated using magnetic fiber drawing 
generate substantial fluid flow. Microfluidics \& Nanofluidics, 2015, 18, 167-174.

[77] Zhang P F, Chen H W, Li L, Liu H L, Liu G, Zhang L W, Zhang D Y, Jiang L. Bioinspired smart peristome surface for temperature - Controlled unidirectional water spreading. ACS Applied Materials \& Interfaces, 2017, 9, 5645-5652.

[78] Li Z M, Zhang D Y, Wang D Y, Zhang L W, Feng L, Zhang X Y. A bioinspired flexible film fabricated by surface-tension-assisted replica molding for dynamic control of unidirectional liquid spreading. ACS Applied Materials \& Interfaces, 2019, 11, 48505-48511.

[79] Zhang X Y, Wang D Y, Zhang Y X, Zhang D Y, Li Z M, Liu H. Bioinspired unidirectional liquid spreading channel Principle, design, and manufacture. Advanced Materials Interfaces, 2020, 7, 1901791.

[80] Li C, Wu L, Yu C, Dong Z, Jiang L. Peristome-mimetic curved surface for spontaneous and directional separation of micro water-in-oil drops. Angewandte Chemie International Edition, 2017, 56, 13623-13628.

[81] Li C X, Li N, Zhang X S, Dong Z C, Chen H W, Jiang L. Uni-directional transportation on peristome-mimetic surfaces for completely wetting liquids. Angewandte Chemie International Edition, 2016, 55, 14988-14992.

[82] Liu X J, Gu H C, Wang M, Du X, Gao B B, Elbaz A, Sun L D, Liao J L, Xiao P F, Gu Z Z. 3D Printing of bioinspired liquid superrepellent structures. Advanced Materials, 2018, 30, e1800103.

[83] Li J Q, Zhou X F, Li J, Che L F, Yao J, McHale G, Chaudhury M K, Wang Z K. Topological liquid diode. Science Advances, 2017, 3, eaao3530.

[84] Chen H W, Zhang L W, Zhang Y, Zhang P F, Zhang D Y, Jiang L. Uni-directional liquid spreading control on a bio-inspired surface from the peristome of Nepenthes alata. Journal of Materials Chemistry A, 2017, 5, 6914-6920.

[85] Ju J, Yao X, Yang S, Wang L, Sun R Z, He Y X, Jiang L. Cactus stem inspired cone-arrayed surfaces for efficient fog collection. Advanced Functional Materials, 2014, 24, 6933-6938.

[86] Yu C M, Cao M Y, Dong Z C, Wang J M, Li K, Jiang L. Spontaneous and directional transportation of gas bubbles on superhydrophobic cones. Advanced Functional Materials, 2016, 26, 3236-3243.

[87] Ma H Y, Cao M Y, Zhang C H, Bei Z L, Li K, Yu C M, Jiang L. Directional and continuous transport of gas bubbles on superaerophilic geometry-gradient surfaces in aqueous environments. Advanced Functional Materials, 2018, 28, 1705091.
[88] Zhang C H, Zhang B, Ma H Y, Li Z, Xiao X, Zhang Y H, Cui X Y, Yu C M, Cao M Y, Jiang L. Bioinspired pressure-tolerant asymmetric slippery surface for continuous self-transport of gas bubbles in aqueous environment. $A C S$ Nano, 2018, 12, 2048-2055.

[89] Zhu H G, Chen D Y, Li N J, Xu Q F, Li H, He J H, Lu J M. Dual-layer copper mesh for integrated oil-Water separation and water purification. Applied Catalysis B: Environmental, 2017, 200, 594-600.

[90] Yong J L, Fang Y, Chen F, Huo J L, Yang Q, Bian H, Du G Q, Hou X. Femtosecond laser ablated durable superhydrophobic PTFE films with micro-through-holes for oil/water separation: Separating oil from water and corrosive solutions. Applied Surface Science, 2016, 389, 1148-1155.

[91] Stogin B B, Gockowski L, Feldstein H, Claure H, Wang J, Wong T S. Free-standing liquid membranes as unusual particle separators. Science Advances, 2018, 4, eaat3276.

[92] Li C X, Dai H Y, Gao C, Wang T, Dong Z C, Jiang L. Bioinspired inner microstructured tube controlled capillary rise. PNAS, 2018, 116, 12704-12709.

[93] Chen H W, Zhang L W, Zhang D Y, Zhang P F, Han Z W. Bioinspired surface for surgical graspers based on the strong wet friction of tree frog toe pads. ACS Applied Materials \& Interfaces, 2015, 7, 13987-13995.

[94] Massarweh N N, Cosgriff N, Slakey D P. Electrosurgery: history, principles, and current and future uses. Journal of the American College of Surgeons, 2006, 202, 520-530.

[95] Han Z W, Fu J, Feng X M, Niu S C, Zhang J Q, Ren L Q. Bionic anti-adhesive electrode coupled with maize leaf microstructures and $\mathrm{TiO}_{2}$ coating. RSC Advances, 2017, 7, 45287-45293.

[96] Liu G, Zhang P F, Liu Y, Zhang D Y, Chen H W. Self-lubricanting slippery surface with wettability gradients for anti-sticking of electrosurgical scalpel. Micromachines (Basel), 2018, 9, 591.

[97] Zhao Z H, Chen H W, Liu X L, Liu H, Zhang D Y. Development of high-efficient synthetic electric heating coating for anti-icing/de-icing. Surface and Coatings Technology, 2018, 349, 340-346.

[98] Liu X L, Chen H W, Zhao Z H, Yan Y Y, Zhang D Y. Slippery liquid-infused porous electric heating coating for anti-icing and de-icing applications. Surface and Coatings Technology, 2019, 374, 889-896.

[99] Hou Y P, Gao L C, Feng S L, Chen Y, Xue Y, Jiang L, Zheng Y M. Temperature-triggered directional motion of tiny water droplets on bioinspired fibers in humidity. Chemical Communications, 2013, 49, 5253-5255. 
[100]Zhu Y Y, Antao D S, Xiao R, Wang E N. Real-time manipulation with magnetically tunable structures. Advanced Materials, 2014, 26, 6442-6446.

[101]Whitby M, Quirke N. Fluid flow in carbon nanotubes and nanopipes. Nature Nanotechnology, 2007, 2, 87-94.

[102]Rossi M P, Ye H H, Gogotsi Y, Babu S, Ndungu P, Bradley J C. Environmental scanning electron microscopy study of water in carbon nanopipes. Nano Letters, 2004, 4, 989-993.

[103]Hummer G, Rasaiah J C, Noworyta J P. Water conduction through the hydrophobic channel of a carbon nanotube. Nature, 2001, 414, 188-190.

[104]Secchi E, Marbach S, Niguès A, Stein D, Siria A, Bocquet L. Massive radius-dependent flow slippage in carbon nanotubes. Nature, 2016, 537, 210-213. 